\title{
Advanced atomic layer deposition and epitaxy processes
}

\section{Citation}

Gordon, Roy G., Xiabing Lou Wang, Sang Bok Kim. 2015. Advanced Atomic Layer Deposition and Epitaxy Processes. 2015. In the Proceedings of the International Symposium on VLSI Technology, Systems and Applications. Hsinchu, Taiwan, April 27-29, 2015.

\section{Published Version}

doi:10.1109/VLSI-TSA.2015.7117593

\section{Permanent link}

http://nrs.harvard.edu/urn-3:HUL.InstRepos:32186504

\section{Terms of Use}

This article was downloaded from Harvard University's DASH repository, and is made available under the terms and conditions applicable to Open Access Policy Articles, as set forth at http:// nrs.harvard.edu/urn-3:HUL.InstRepos:dash.current.terms-of-use\#OAP

\section{Share Your Story}

The Harvard community has made this article openly available.

Please share how this access benefits you. Submit a story.

Accessibility 
2015 International Symposium on

VLSI Technology, Systems and Applications

\section{Advanced Atomic Layer \\ Deposition and Epitaxy Processes}

Roy Gordon

Professor of Chemistry and Materials Science

Harvard University

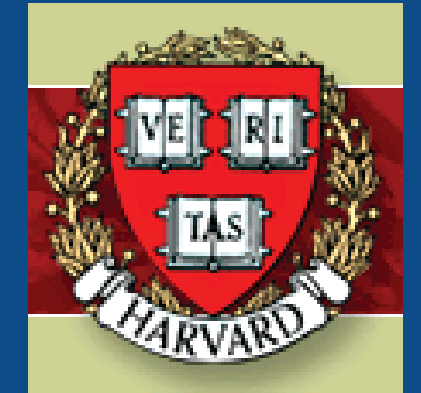




\section{Single-crystal oxide insulators grown epitaxially on GaAs, Ge and GaN by ALE}

\section{Outline}

Atomic Layer Epitaxy (ALE)

ALE of single-crystal $\mathrm{La}_{2} \mathrm{O}_{3}$ on $\mathrm{GaAs}(111)$

CMOS circuits with $\mathrm{La}_{2} \mathrm{O}_{3}$ on $\mathrm{GaAs}(111)$

ALE of single-crystal $\mathrm{La}_{2} \mathrm{O}_{3}$ on $\mathrm{Ge}(111)$

ALE of single-crystal (Mg, Ca)O on GaN(0001) 


\section{ITRS roadmap}

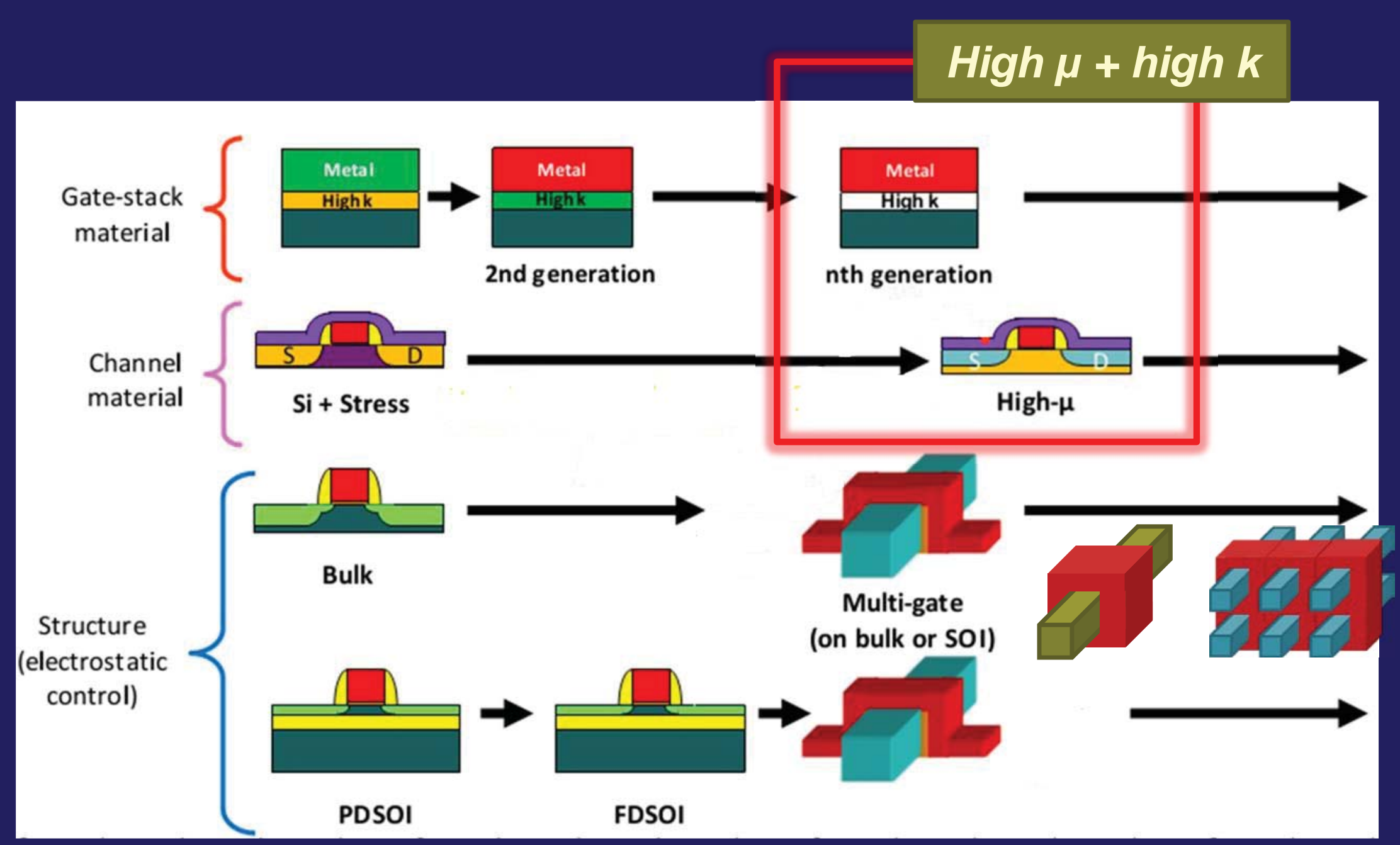




\section{GaAs has Many Traps at Oxide Interfaces}
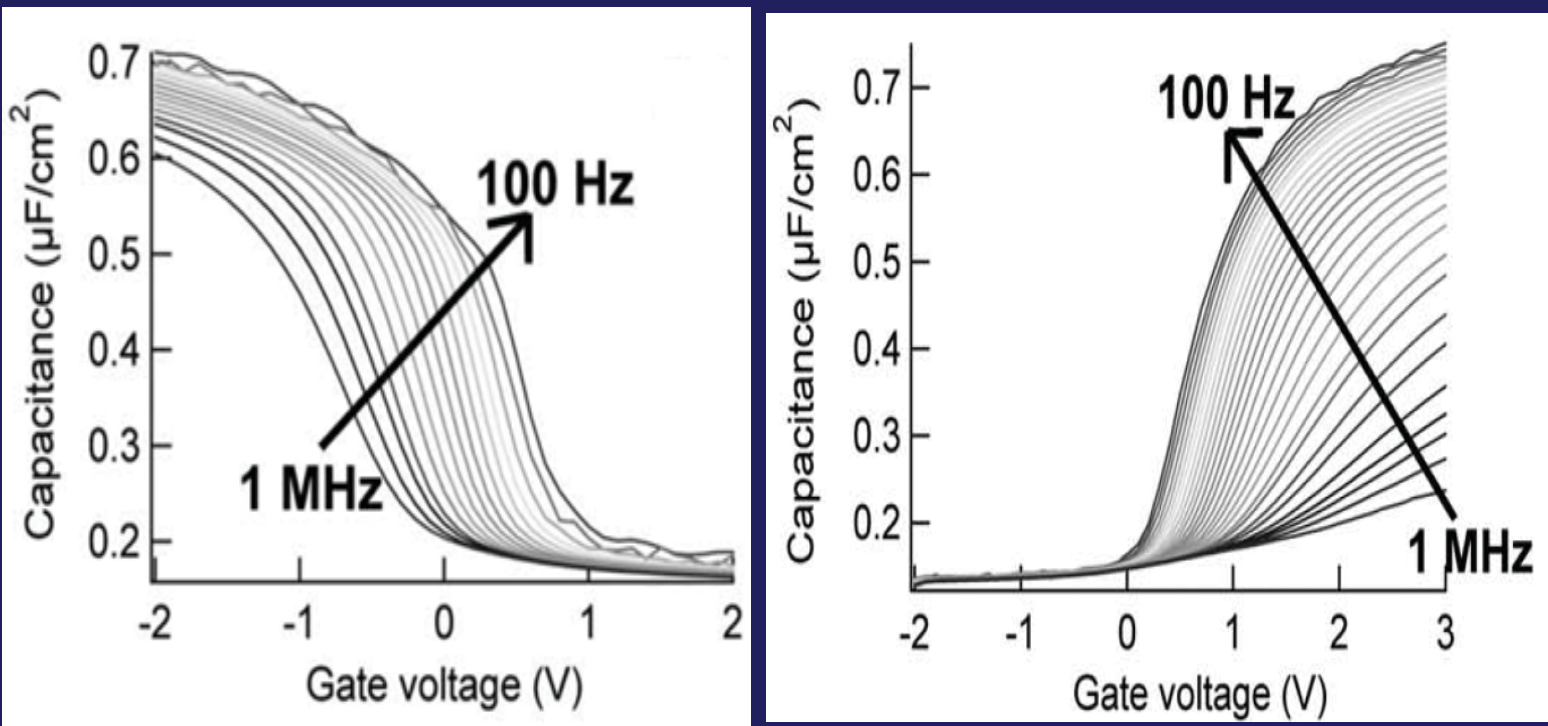

=> very large frequency dispersion

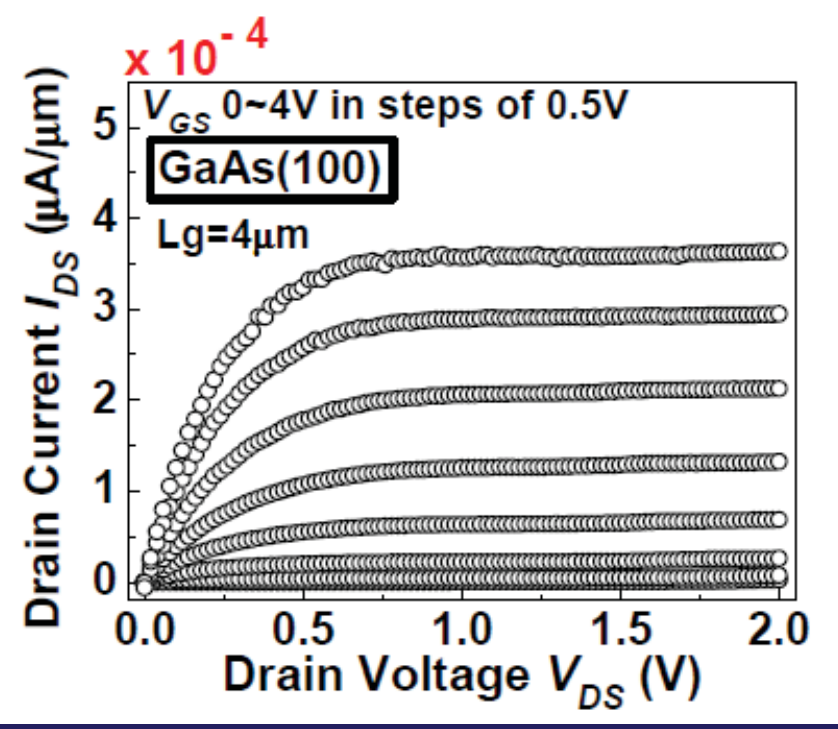

=> very low drive currents

$\sim 10^{13} \mathrm{~cm}^{-2}$ traps at GaAs interface with oxides $<10^{11} \mathrm{~cm}^{-2}$ traps at $\mathrm{Si} / \mathrm{SiO}_{2}$ interface

$\Rightarrow$ GaAs MOS transistors are much poorer than $\mathrm{Si}$

Solution: epitaxial single-crystal $\mathrm{La}_{2} \mathrm{O}_{3}$ on GaAs

$\Rightarrow \sim 10^{11} \mathrm{~cm}^{-2}$ traps at $\mathrm{GaAs} / \mathrm{La}_{2} \mathrm{O}_{3}$ interface

$\Rightarrow$ First CMOS transistors ever made on GaAs 


\section{Atomic Layer Epitaxy (ALE)}

Sequential, self-limiting surface reactions make alternating layers:
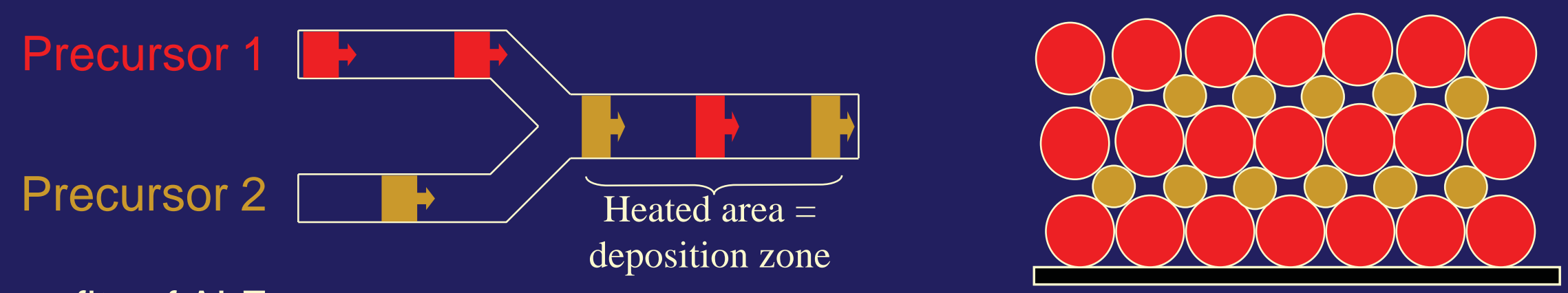

Benefits of ALE:

- High-quality epitaxial interfaces with few traps

- Atomic level of control over film composition

$\Rightarrow$ nanolaminates and multi-component materials

- Uniform thickness over large areas and inside narrow holes

- Smooth surfaces

- High density and few defects or pinholes

- Low deposition temperatures (for very reactive precursors)

- Pure films (for suitably reactive precursors)

- Full-wafer semiconductor-grade production equipment available 


\section{Amidinate Precursors for Trivalent Metals}

La, Ce, Pr, Nd, Sm, Eu, Gd, Tb, Dy, Ho, Er, Tm, Yb, Lu, Y, Sc, In, Ru, Ti

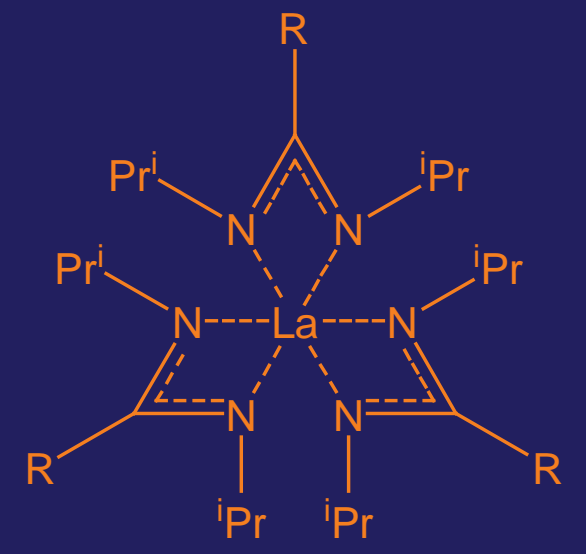

$\mathrm{R}=\mathrm{H}$ (formamidinate, $\mathrm{fmd}$ )

$\mathrm{R}=\mathrm{CH}_{3}$ (acetamidinate, amd)

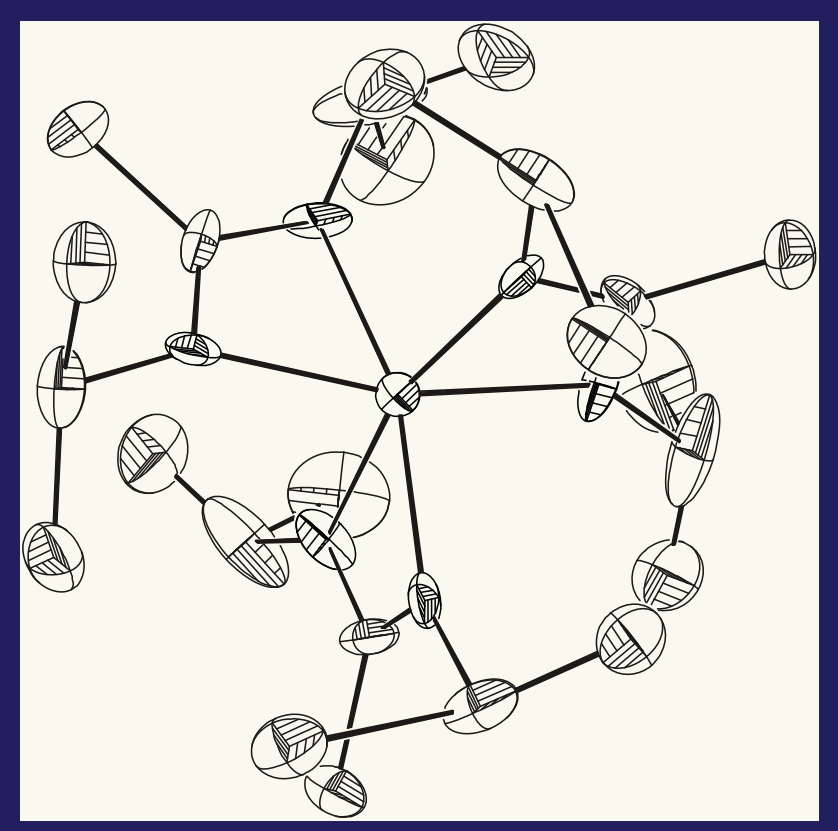

Now produced commercially by the Dow Chemical Company 


\section{Vaporization of Lanthanum Amidinate Precursor}

tris(N,N'-diisopropyl-formamidinato)lanthanum $\mathrm{La}\left(\left(\mathrm{Pr}_{2} \mathrm{~N}\right)_{2} \mathrm{CH}\right)_{3}$ abbreviated $\mathrm{La}(\mathrm{fmd})_{3}$
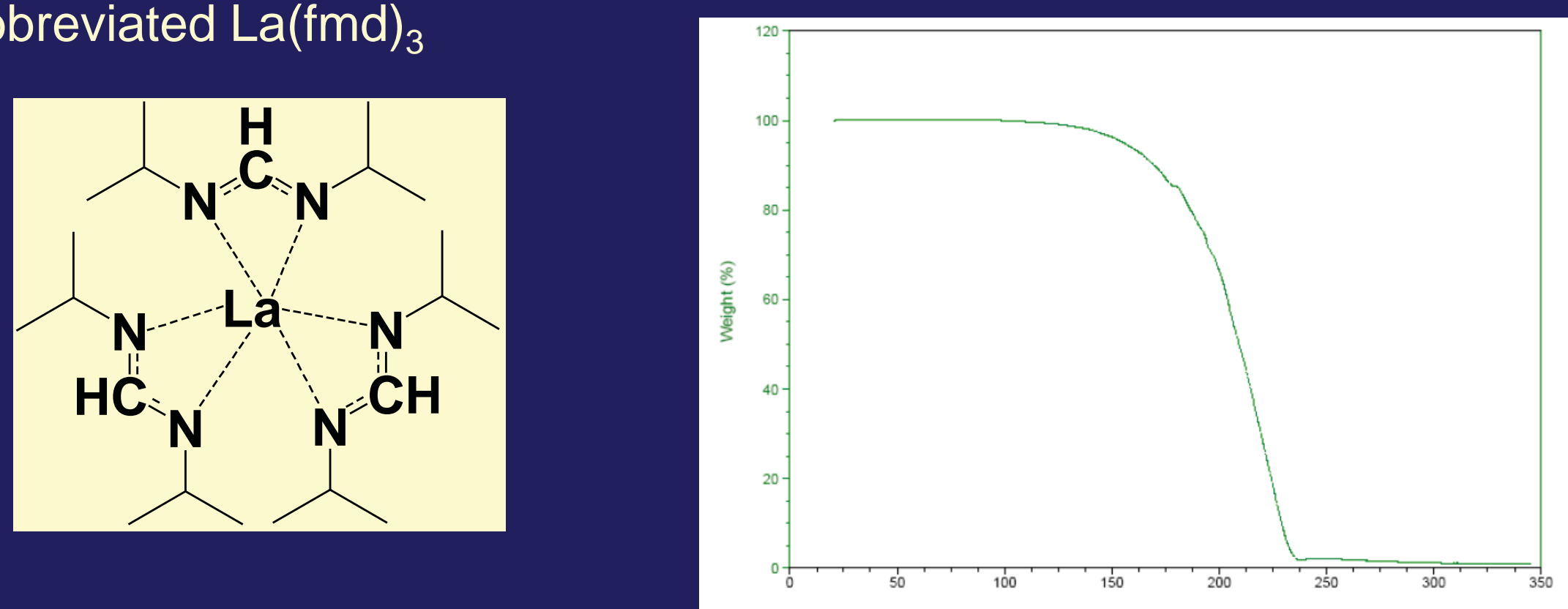

Most volatile lanthanum compound known

Complete evaporation without decomposition or residue 


\section{ALE of Lanthanum Oxide, $\mathrm{La}_{2} \mathrm{O}_{3}$}

Precursors: $\mathrm{H}_{2} \mathrm{O}$ and $\mathrm{La}(\mathrm{fmd})_{3}$

$\mathrm{La}(\mathrm{fmd})_{3}$ at $120^{\circ} \mathrm{C}$, substrate at $300^{\circ} \mathrm{C}$
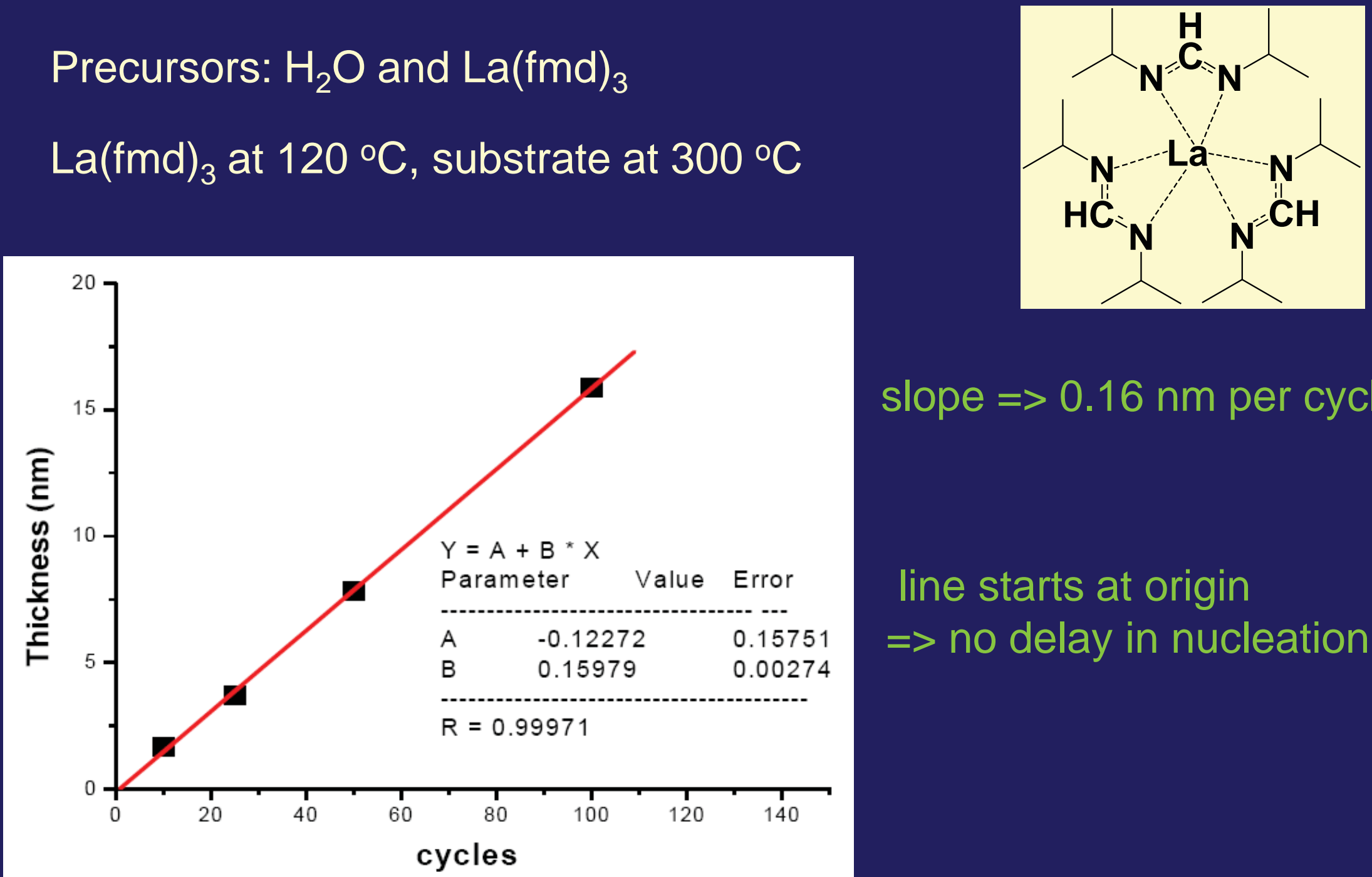

slope $=>0.16 \mathrm{~nm}$ per cycle

line starts at origin

$=>$ no delay in nucleation 


\section{ALE $\mathrm{La}_{2} \mathrm{O}_{3}$ is Epitaxial on $\mathrm{GaAs}(111) \mathrm{A}$}

TEM

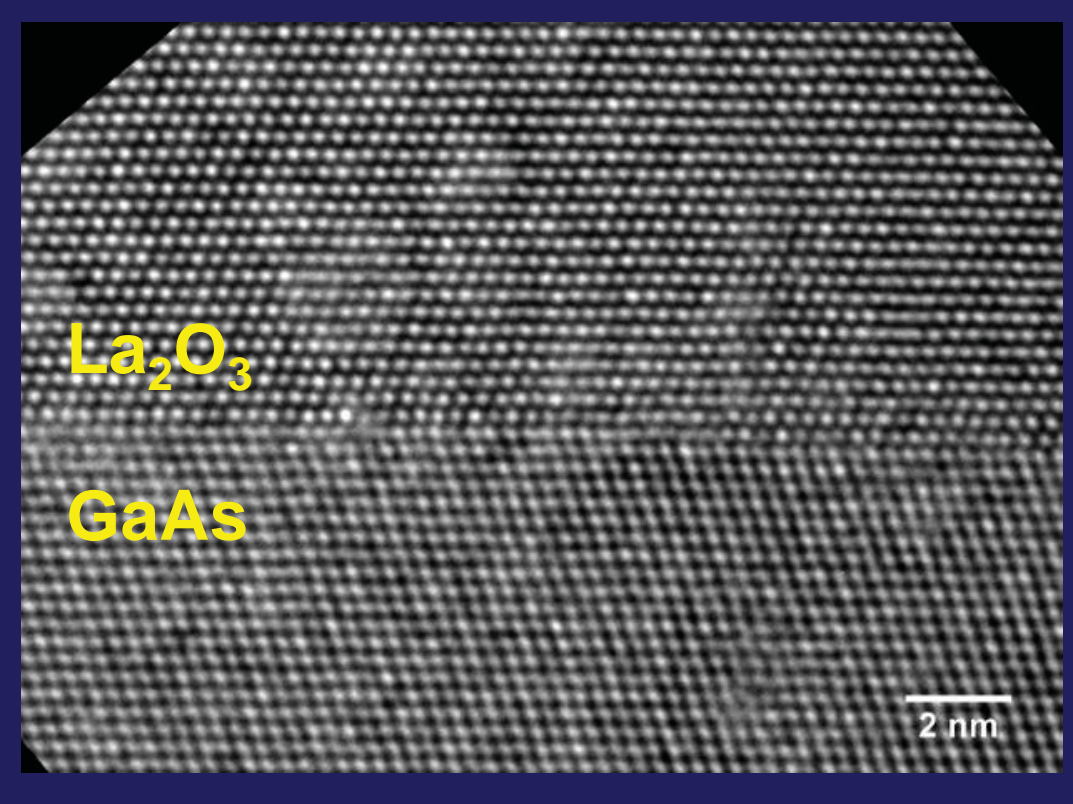

Lattice Constant Matching: $\mathrm{a}\left(\mathrm{La}_{2} \mathrm{O}_{3}\right)=2.0008 \times \mathrm{a}(\mathrm{GaAs})$ or $0.04 \%$ mismatch
High Resolution XRD

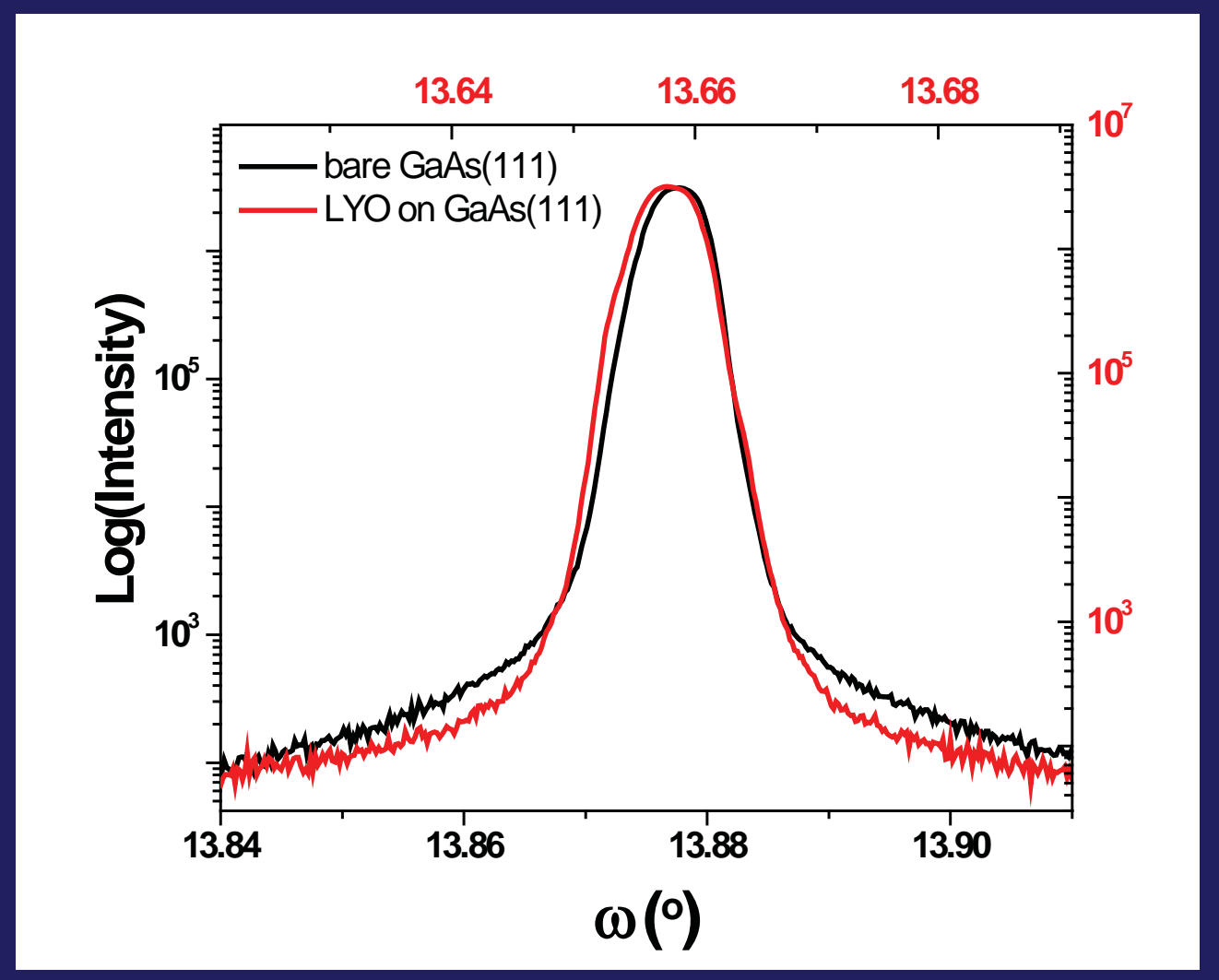




\section{Atomic layer epitaxy of $\mathrm{GaAs}(111) / \mathrm{La}_{2} \mathrm{O}_{3}$}

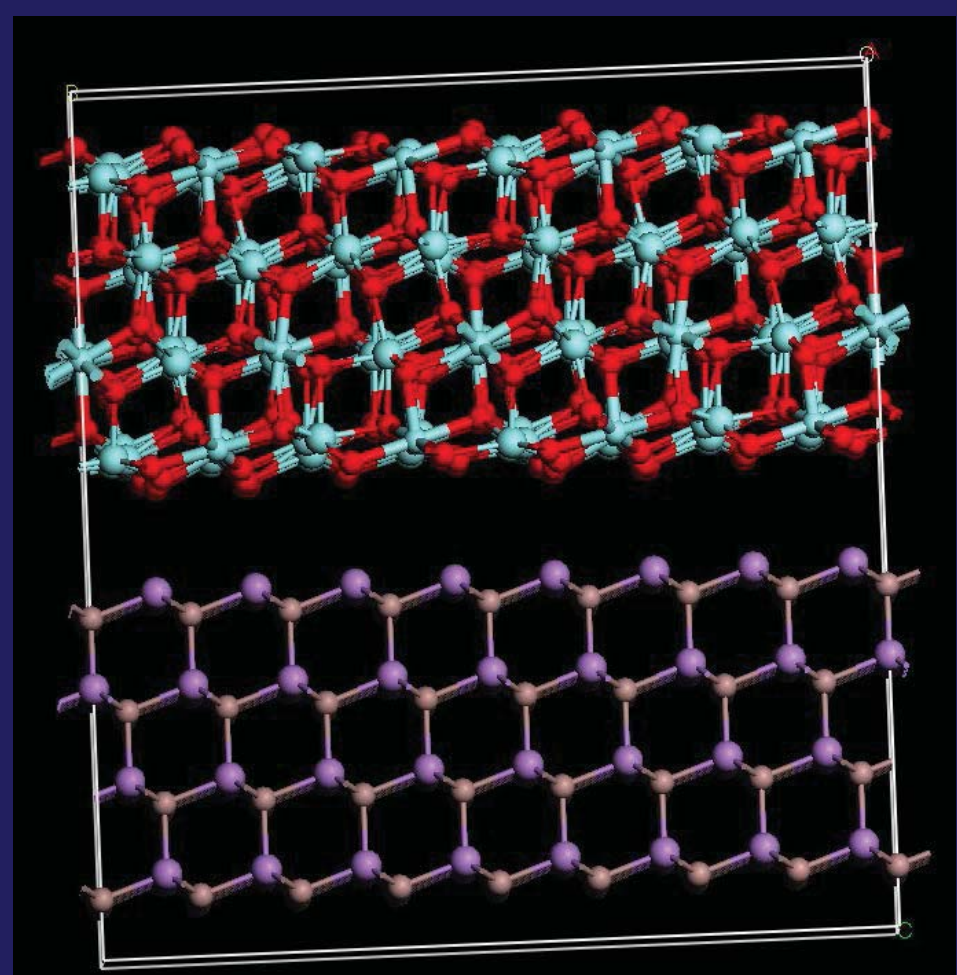

Side view of $\mathrm{GaAs}(111) \mathrm{A} \mathrm{La}_{2} \mathrm{O}_{3}$
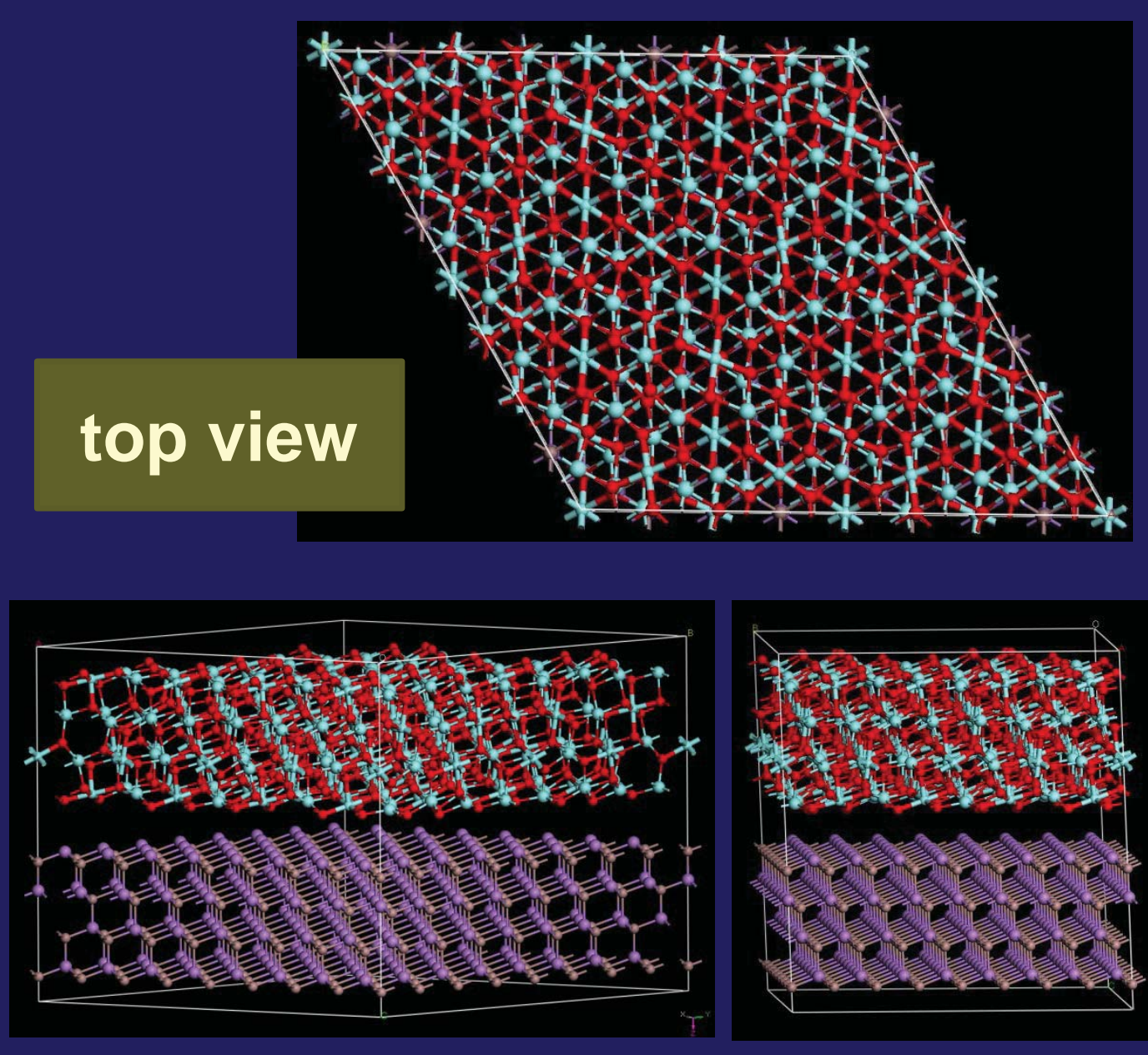


\section{CV characteristics of GaAs(111)/La $2-\mathrm{x} \mathrm{Y}_{\mathrm{x}} \mathrm{O}_{3}$}

Closer Lattice Match to GaAs $\Rightarrow>$ less dispersion

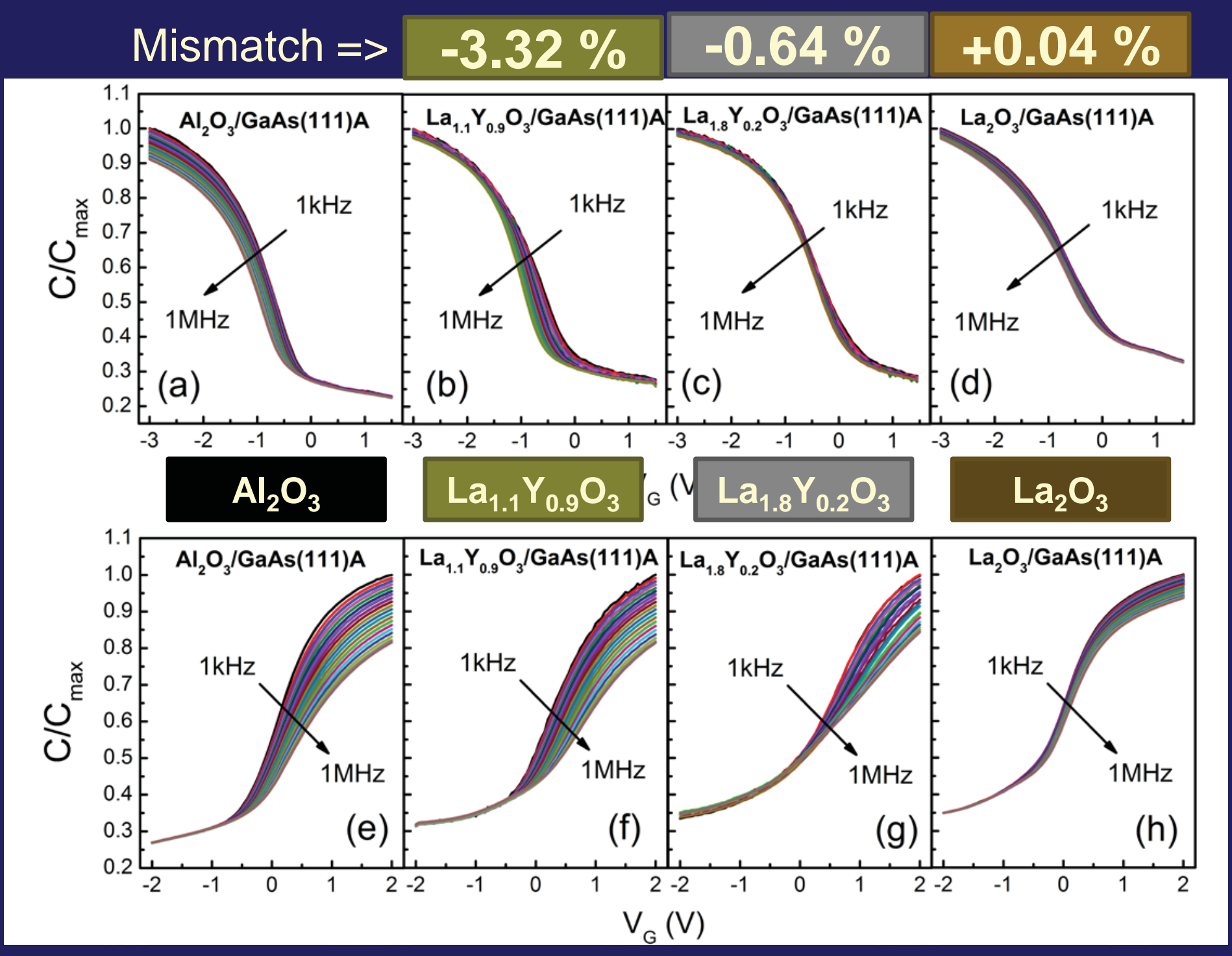




\section{Interface Trap Densities}

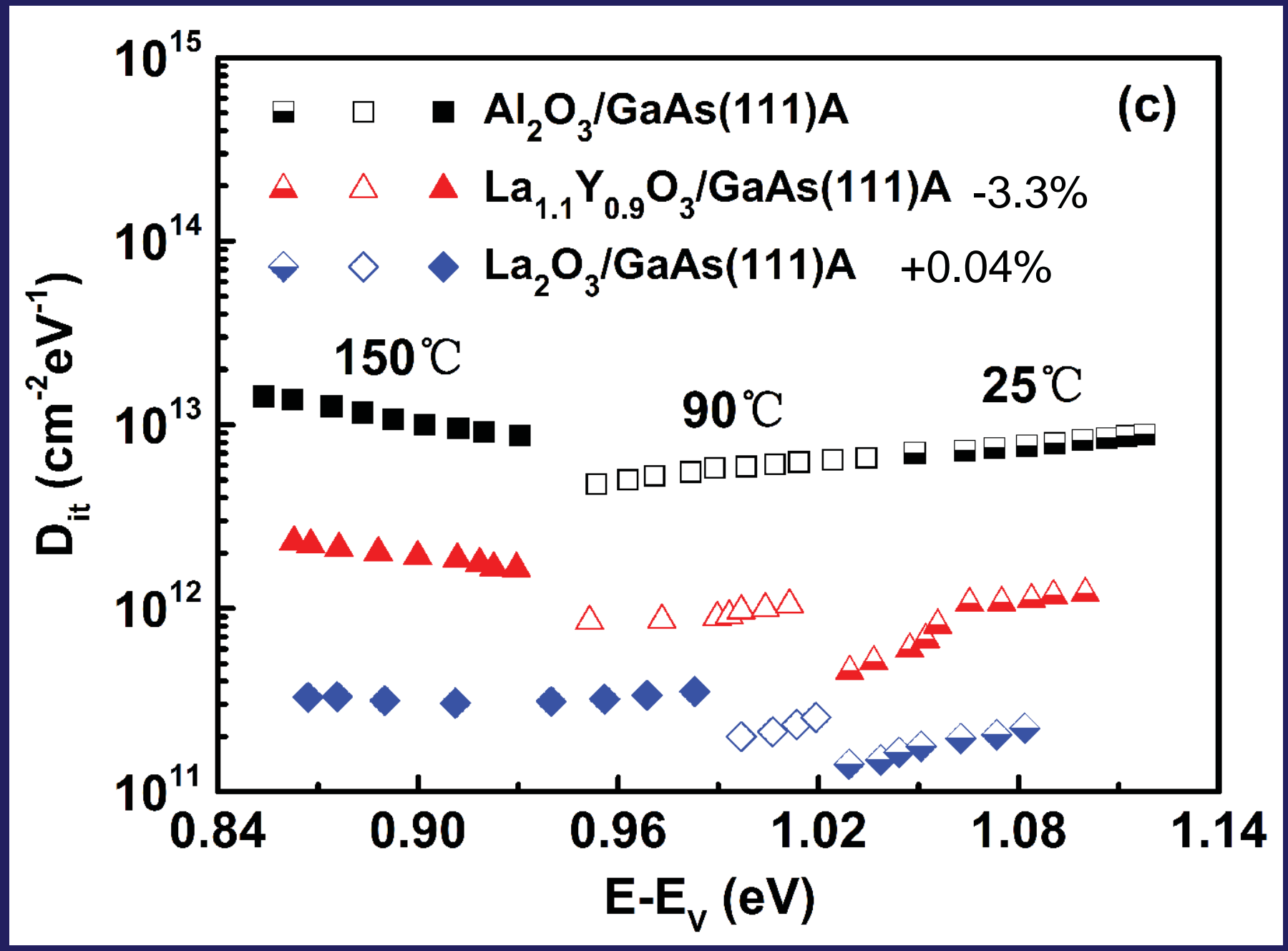




\section{Closer Lattice Match to GaAs => Fewer Traps}

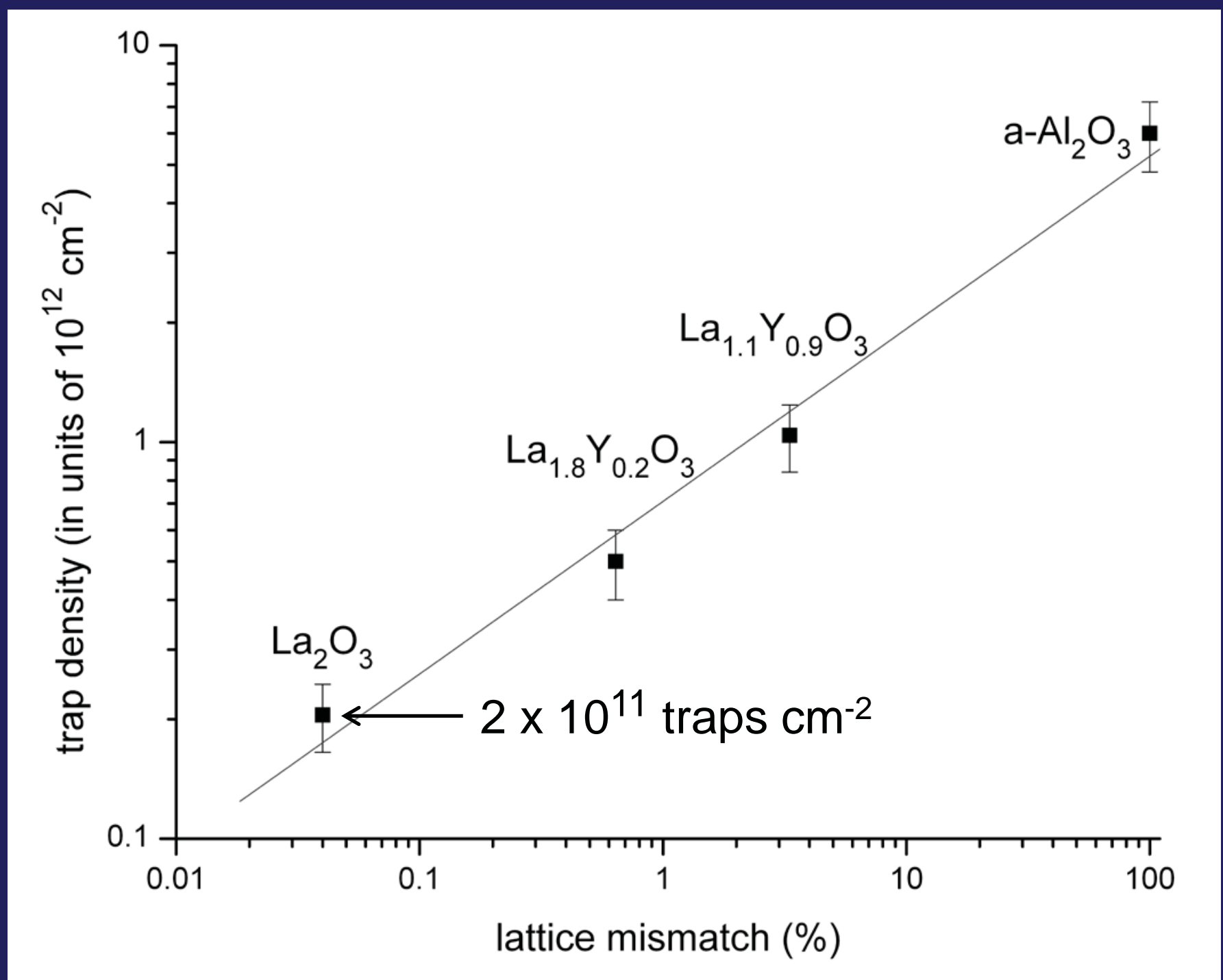




\section{Summany of GaAs CMOS}

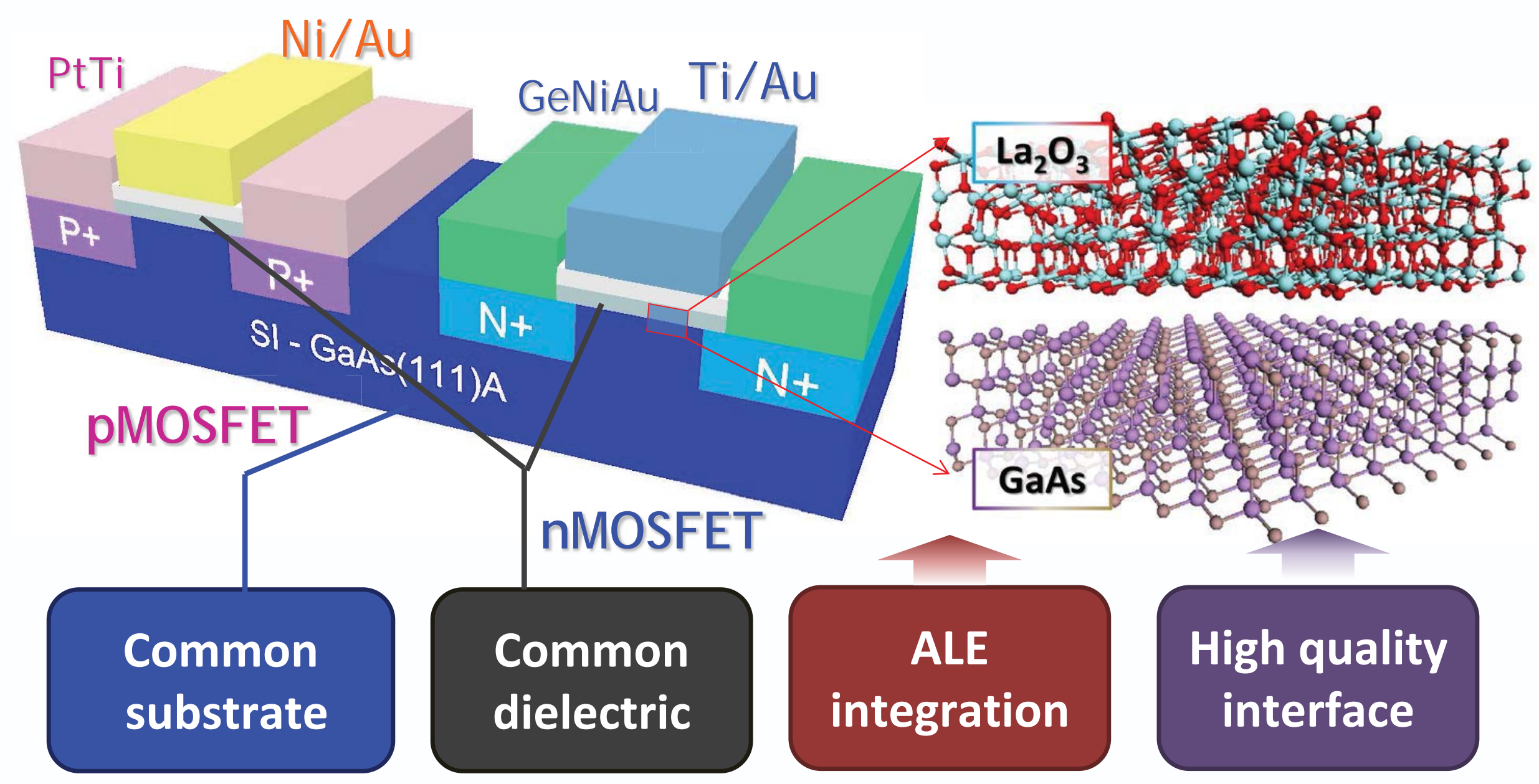




\section{GaAs nMOSFET with $\mathrm{La}_{2} \mathrm{O}_{3}$ epitaxial dielectrics}
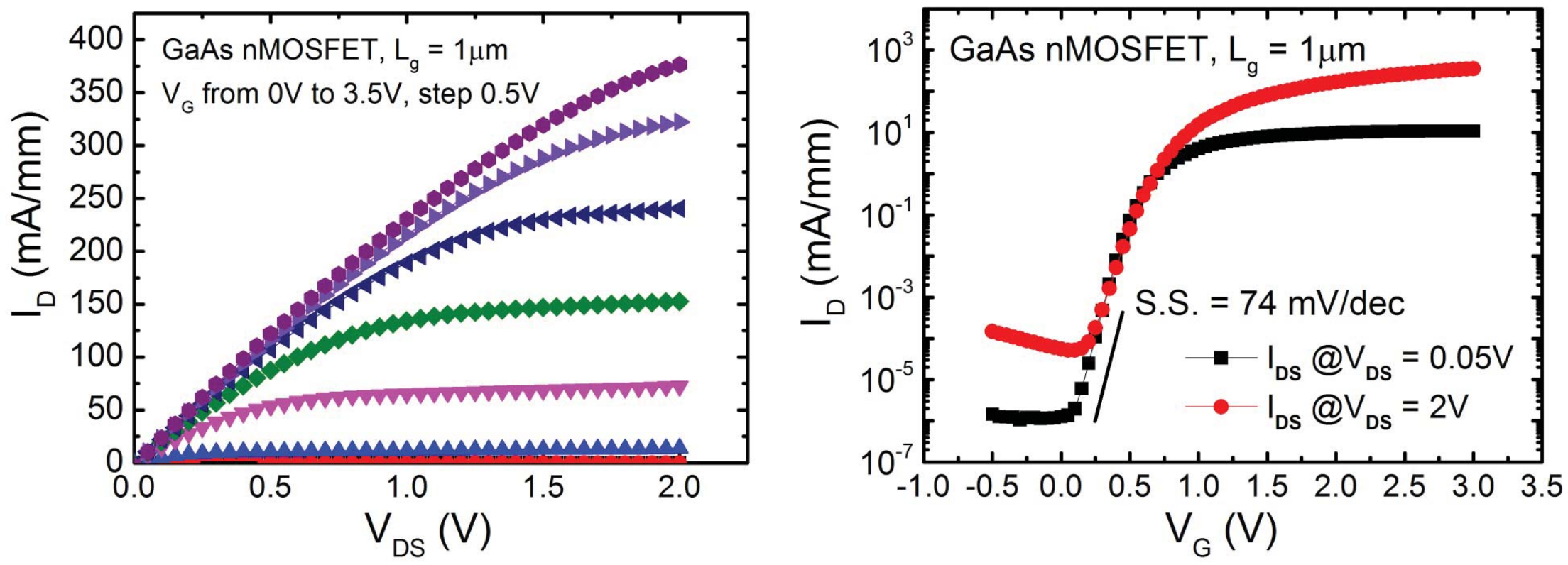

$\square$ High drain current of $376 \mathrm{~mA} / \mathrm{mm}$ is obtained from GaAs nMOSFET with $1 \mu \mathrm{m}$ gate length

$\square$ Current $I_{\text {on }} / I_{\text {off }} \sim 10^{7}$, subthreshold slope as low as $74 \mathrm{mV} / \mathrm{dec}$ $\square$ A mean $D_{\text {it }}$ of $1.7 \times 10^{12} \mathrm{~cm}^{-2} \cdot \mathrm{eV}^{-1}$, using $S S=60\left(1+\mathrm{q} D_{\text {it }} / \mathrm{C}_{\mathrm{ox}}\right)$ 


\section{GaAs nMOSFET with $\mathrm{La}_{2} \mathrm{O}_{3}$ epitaxial dielectric}
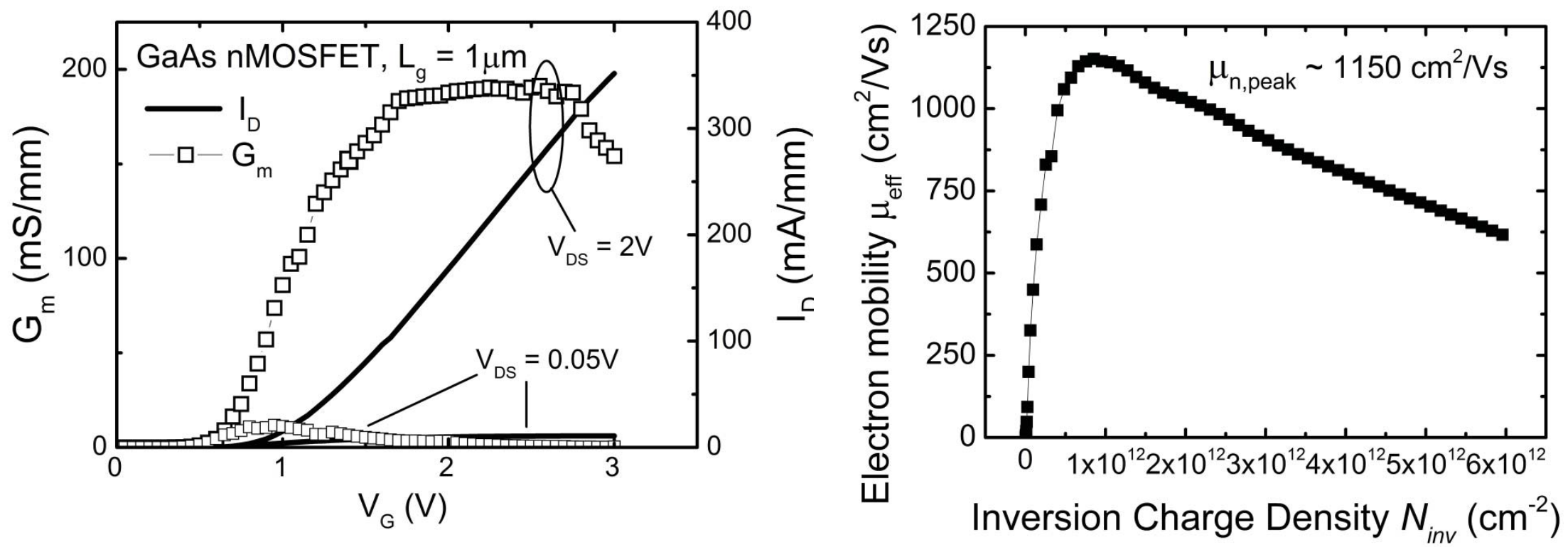

$\square$ Peak transconductance $G_{m} \sim 190 \mathrm{~ms} / \mathrm{mm}$

$\square$ Peak electron effective mobility $\sim 1150 \mathrm{~cm}^{2} / \mathrm{Vs}$ 


\section{GaAs pMOSFET with $\mathrm{La}_{2} \mathrm{O}_{3}$ epitaxial dielectric}
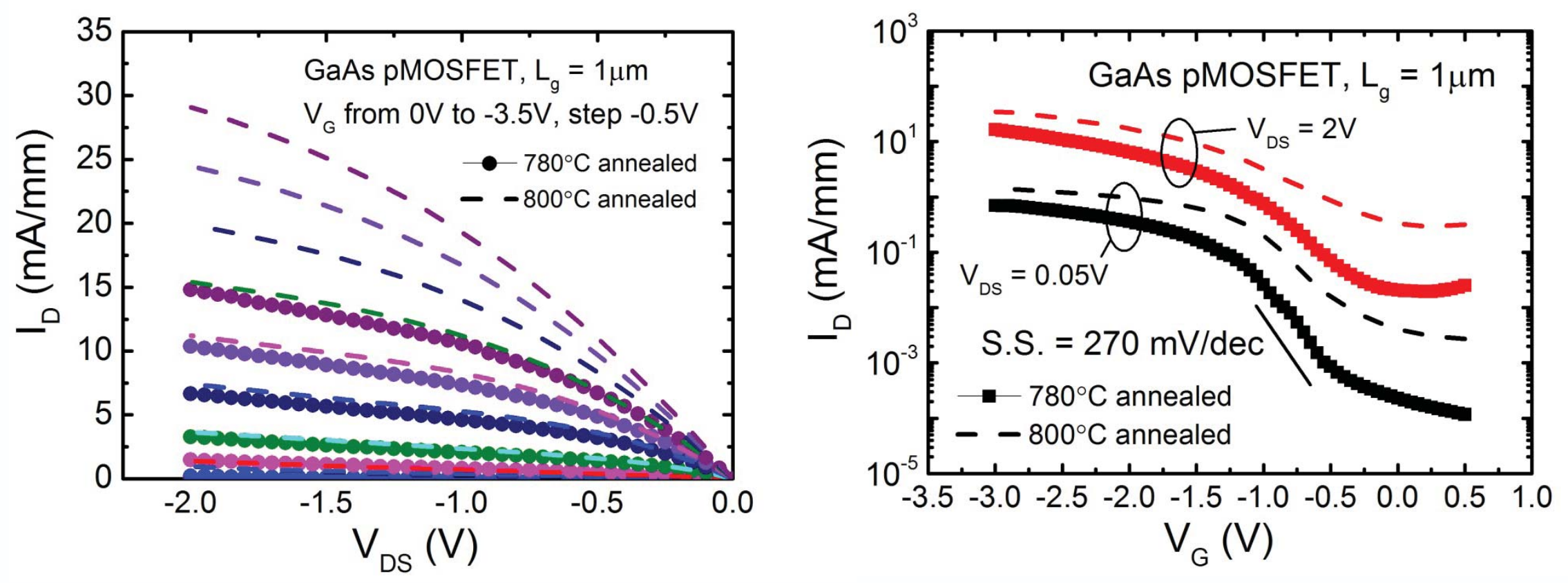

$\square$ Maximum drain current of $30 \mathrm{~mA} / \mathrm{mm}$ is obtained from GaAs pMOSFET with 1 um gate length

$\square I_{\text {on }} / I_{\text {off }} \sim 10^{4}$ and $\sim 10^{3}$ for the pMOSFETs with $1 \mu \mathrm{m}$ gate length annealed at $780^{\circ} \mathrm{C}$ and $800^{\circ} \mathrm{C}$, respectively 


\section{GaAs pMOSFET with $\mathrm{La}_{2} \mathrm{O}_{3}$ epitaxial dielectric}
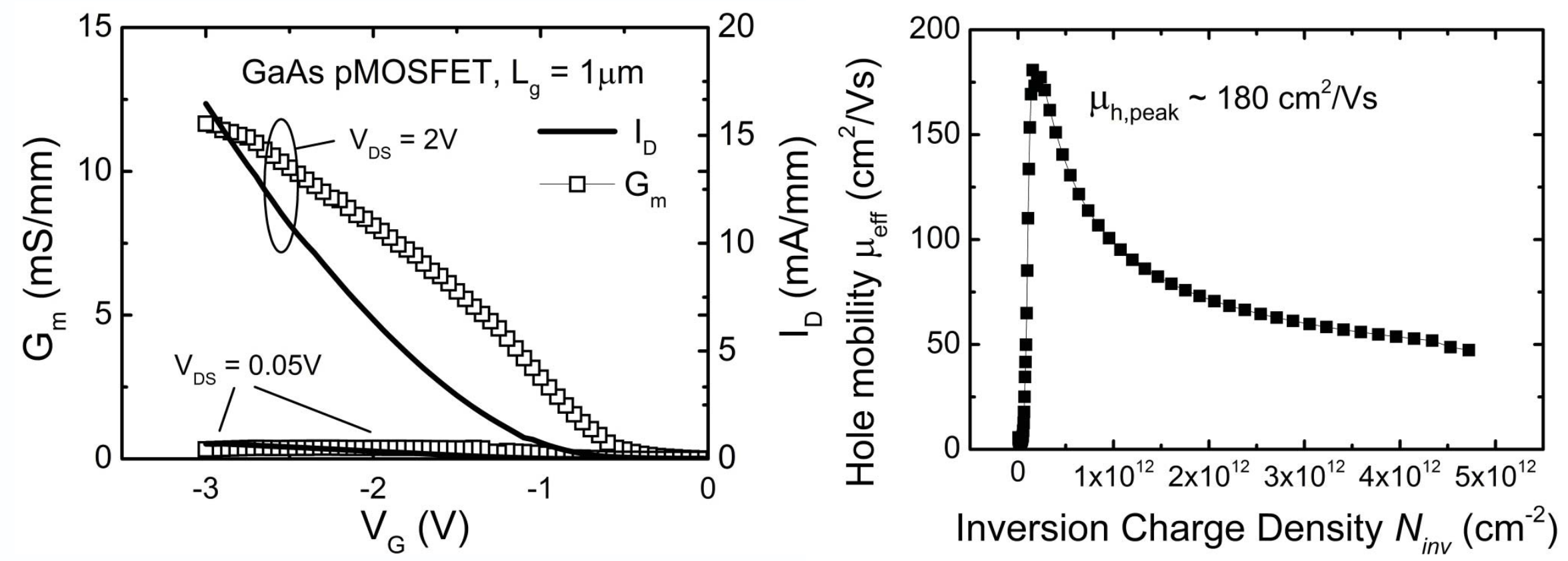

$\square$ A transconductance of $\mathbf{1 2} \mathrm{ms} / \mathrm{mm}$ is obtained from GaAs pMOSFET with $\mathrm{Lg}=1 \mu \mathrm{m}$

$\square$ The peak effective hole mobility is around $180 \mathrm{~cm}^{2} / \mathrm{Vs}$. 


\section{CMOS GaAs inverters}
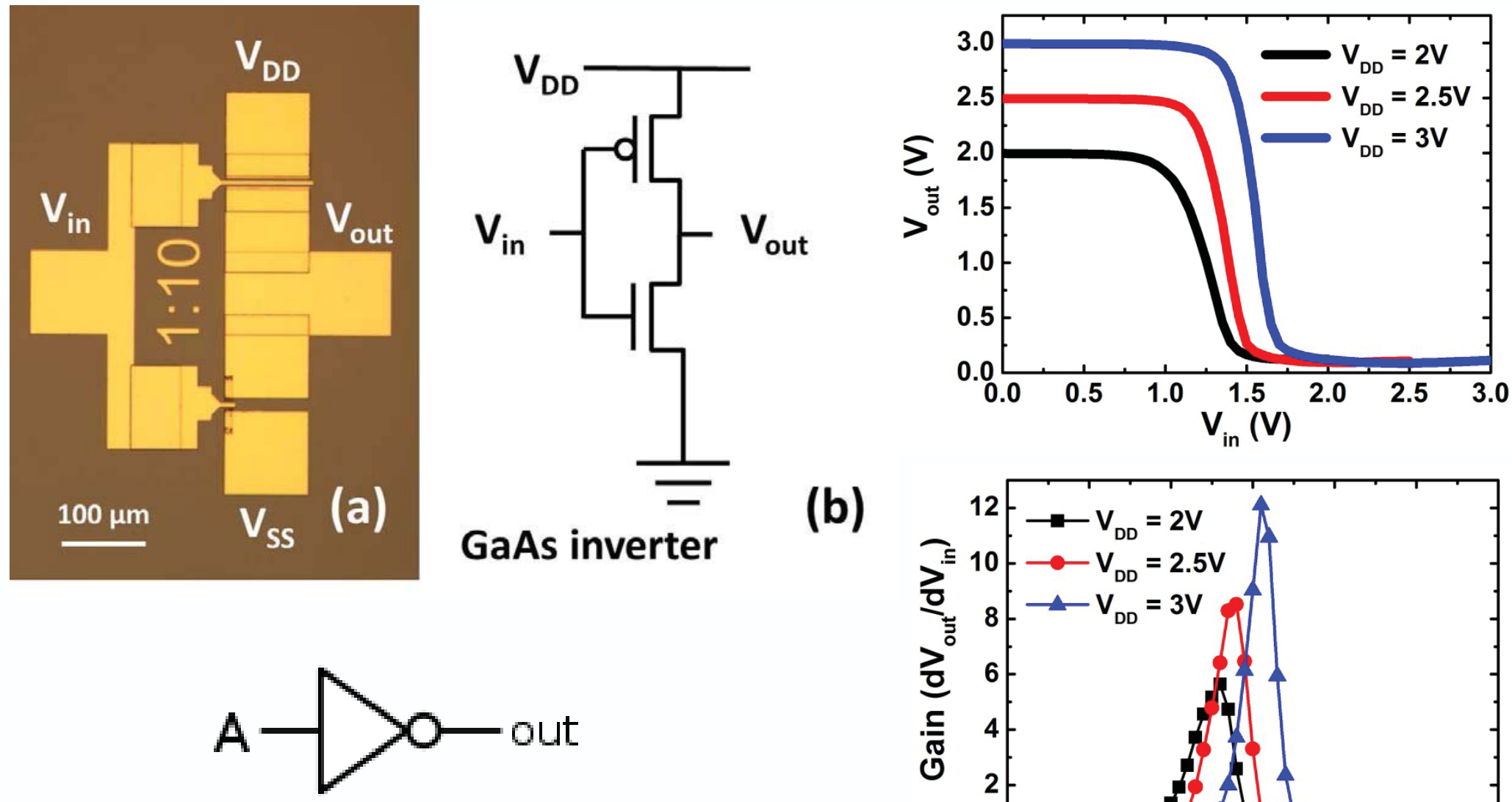

(b)

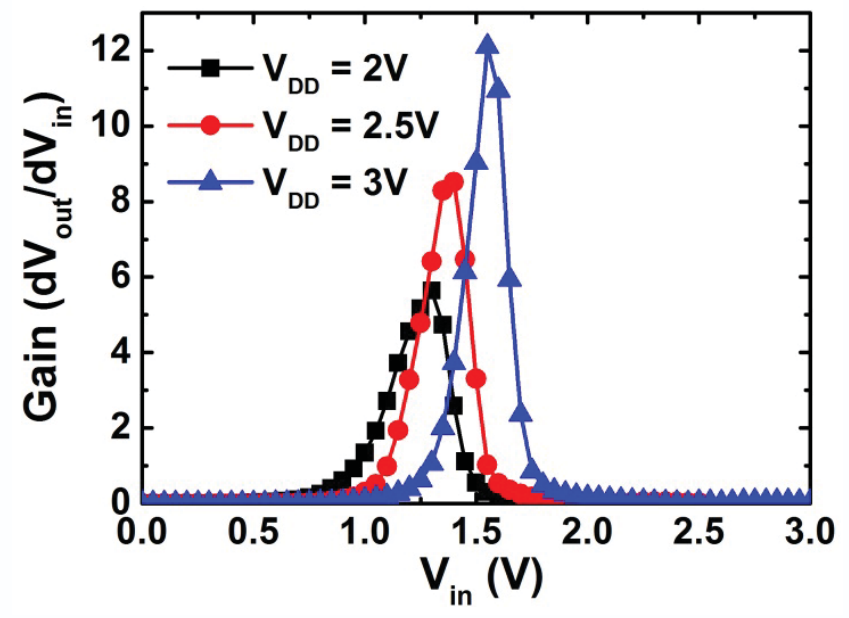

$\square$ Peak gain of 12 is obtained with $V_{D D}=3 V$ 


\section{CMOS GaAs NAND logic gate}
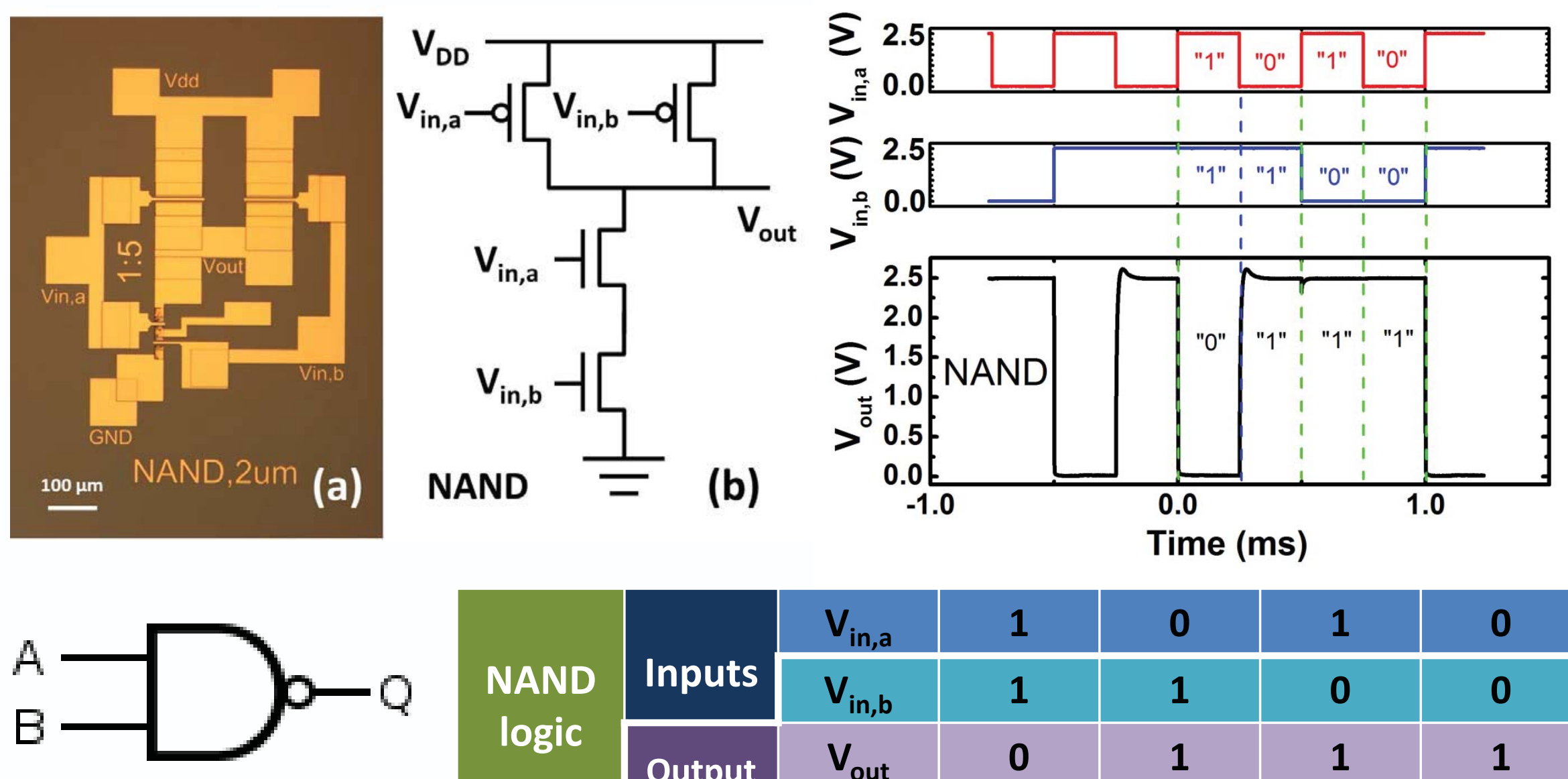

\begin{tabular}{c|c|c|c|c|c|c|}
\multirow{2}{*}{ NAND } & Inputs & $\mathbf{V}_{\text {in,a }}$ & 1 & 0 & 1 & 0 \\
\cline { 2 - 7 } $\operatorname{logic}$ & $\mathbf{V}_{\text {in, }}$ & 1 & 1 & 0 & 0 \\
\cline { 2 - 7 } & Output & $\mathbf{V}_{\text {out }}$ & 0 & 1 & 1 & 1 \\
\hline
\end{tabular}




\section{CMOS GaAs NOR logic gate}
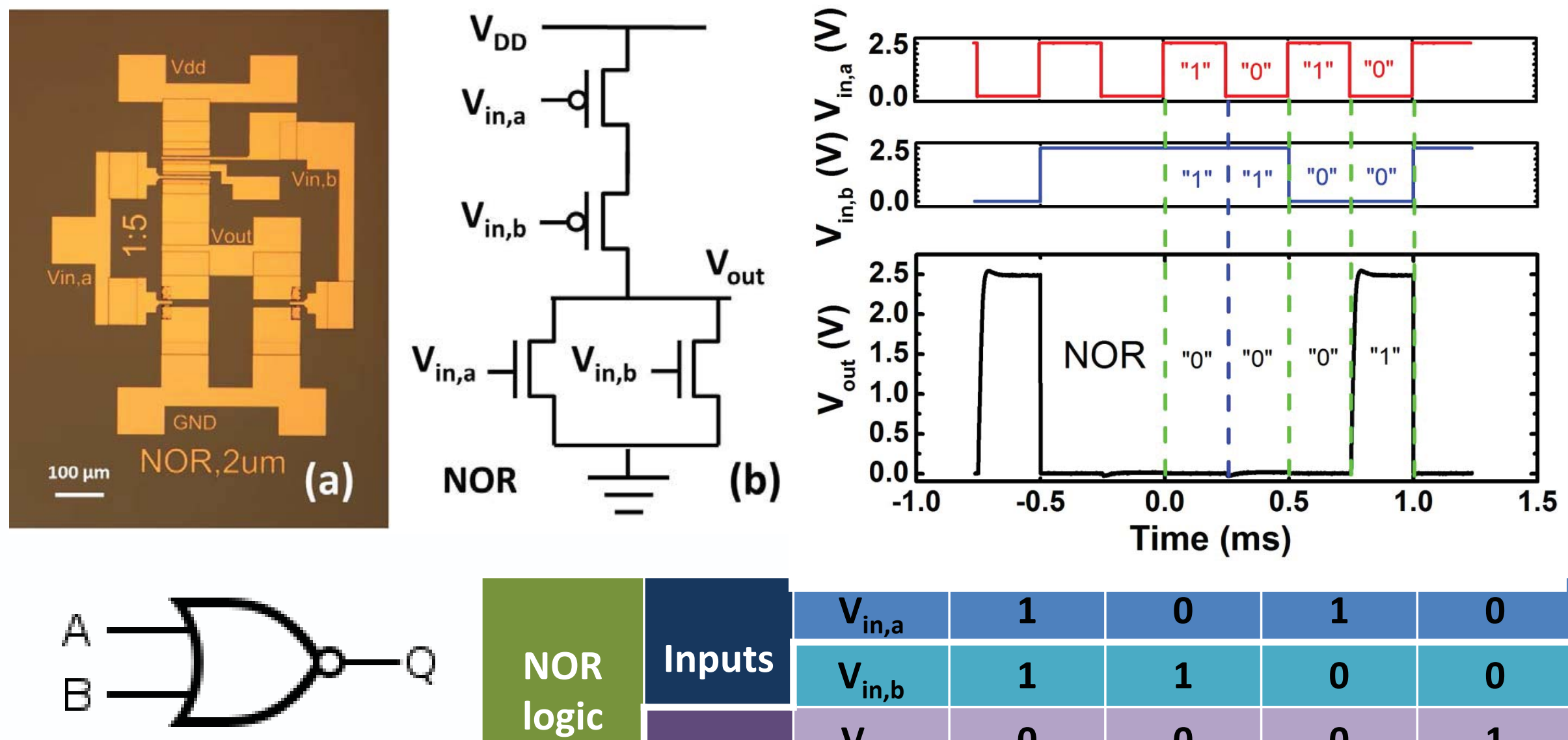

\begin{tabular}{l|l|l|l|l|l|l|}
\multirow{2}{*}{ NOR } & Inputs & $\mathbf{V}_{\text {in,a }}$ & 1 & 0 & 1 & 0 \\
\cline { 2 - 7 } logic & $\mathbf{V}_{\text {in, }}$ & 1 & 1 & 0 & 0 \\
\cline { 2 - 7 } & Output & $\mathbf{V}_{\text {out }}$ & 0 & 0 & 0 & 1 \\
\hline
\end{tabular}




\section{CMOS GaAs 5-stage ring oscillator}

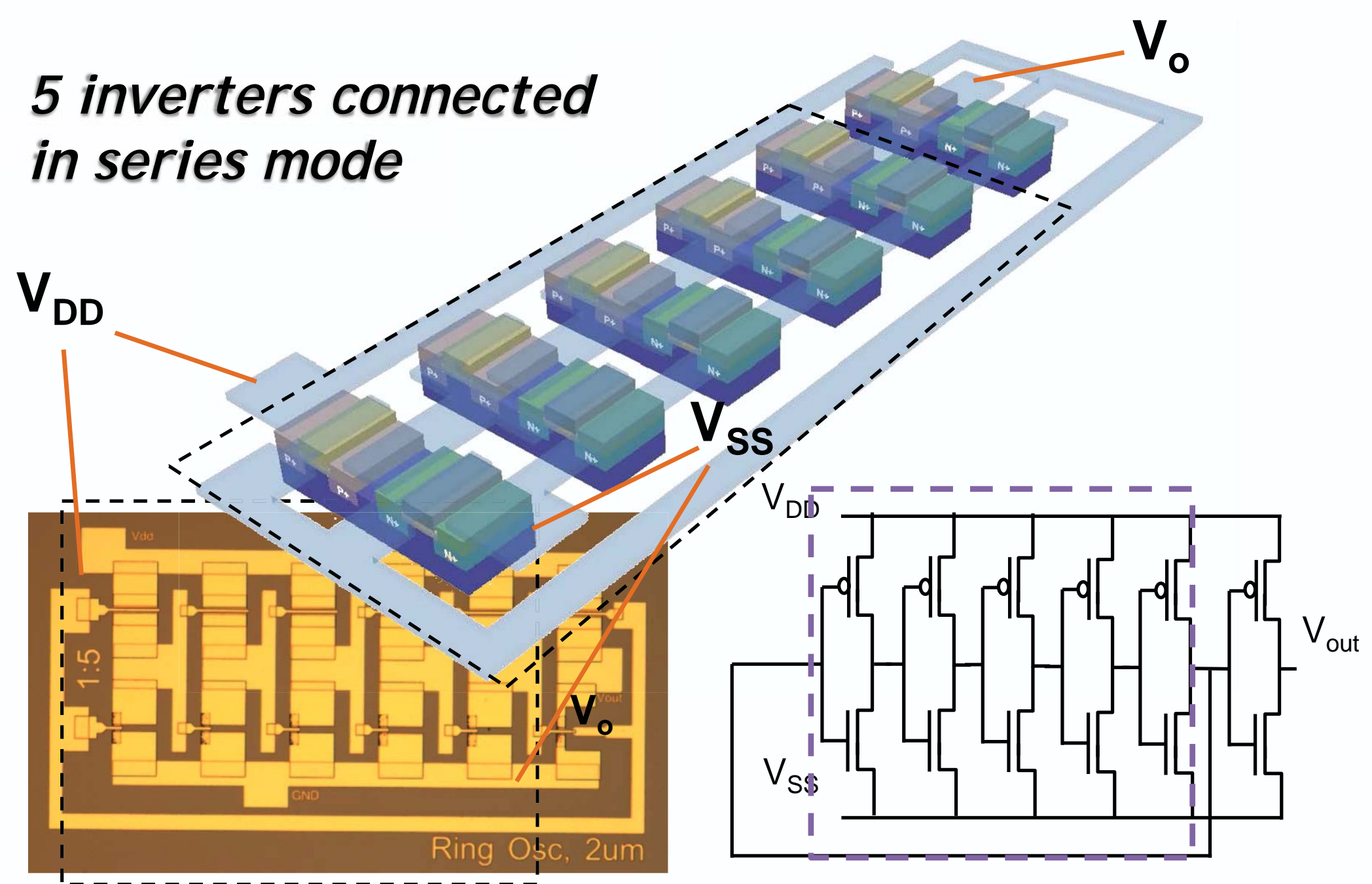




\section{CMOS GaAs 5-stage ring oscillator}
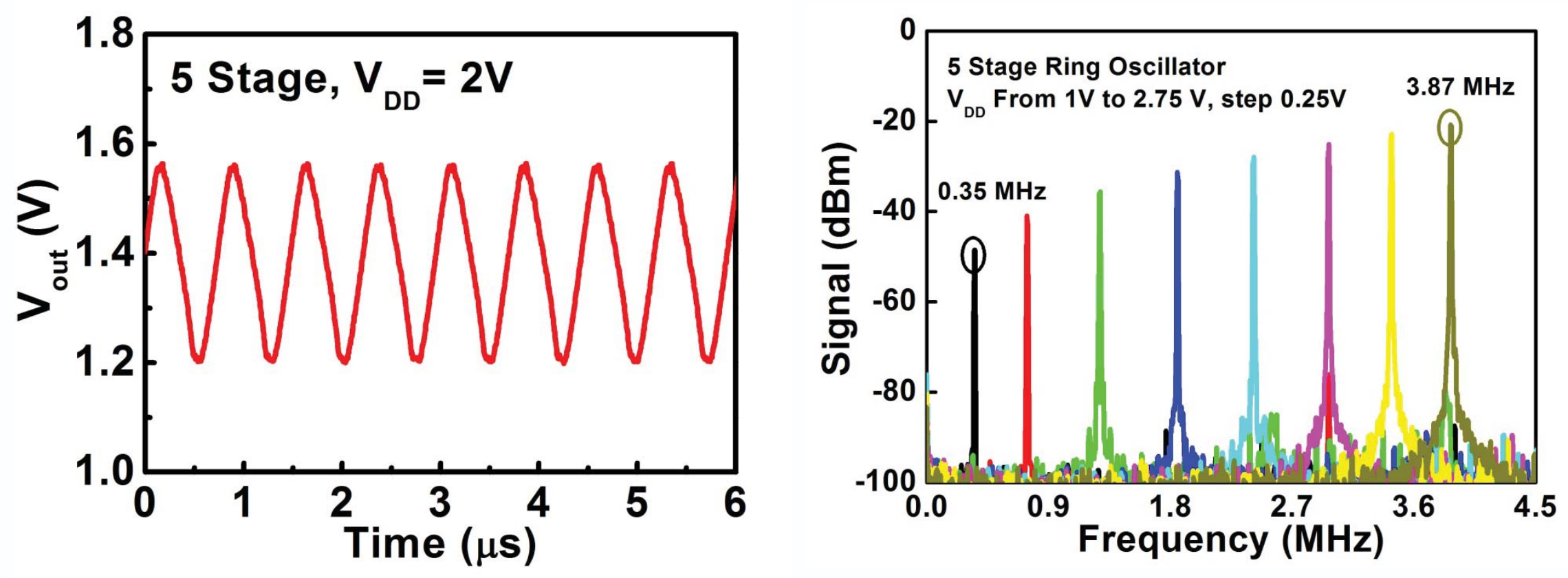

$\square A t V_{D D}=2 \mathrm{~V}$, the fundamental oscillation frequency is at $2.2 \mathrm{MHz}$

$\square$ The fundamental resonance frequency increases from $0.35 \mathrm{Mtz}$ at $\mathrm{V}_{\mathrm{DD}}=1 \mathrm{~V}$ to $3.87 \mathrm{MH}$ at $\mathrm{V}_{\mathrm{DD}}=2.75 \mathrm{~V}$. 


\section{How to Translate these Results to GaAs(100)}

ALE $\mathrm{La}_{2} \mathrm{O}_{3}$ and $\mathrm{LaLuO}_{3}$ are polycrystalline, not epitaxial, on $\mathrm{GaAs}(100)$

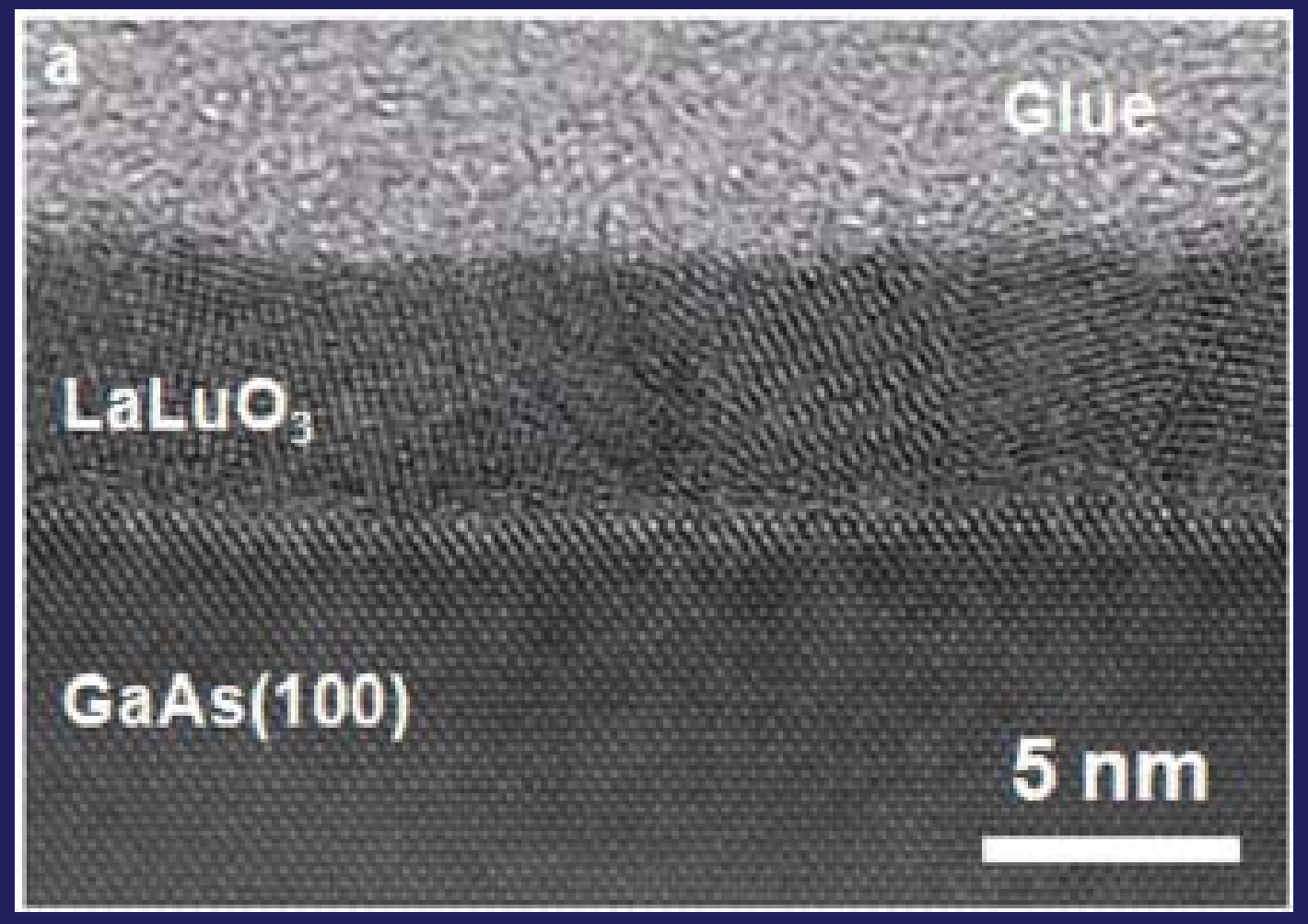

Electrical measurements of capacitors show many traps at interface 


\section{Wet Etching GaAs(100) Exposes (111) Surface}

$=>$ epitaxial $\mathrm{La}_{2} \mathrm{O}_{3}$ on $\mathrm{GaAs}(111)$ facets

\section{(a)}

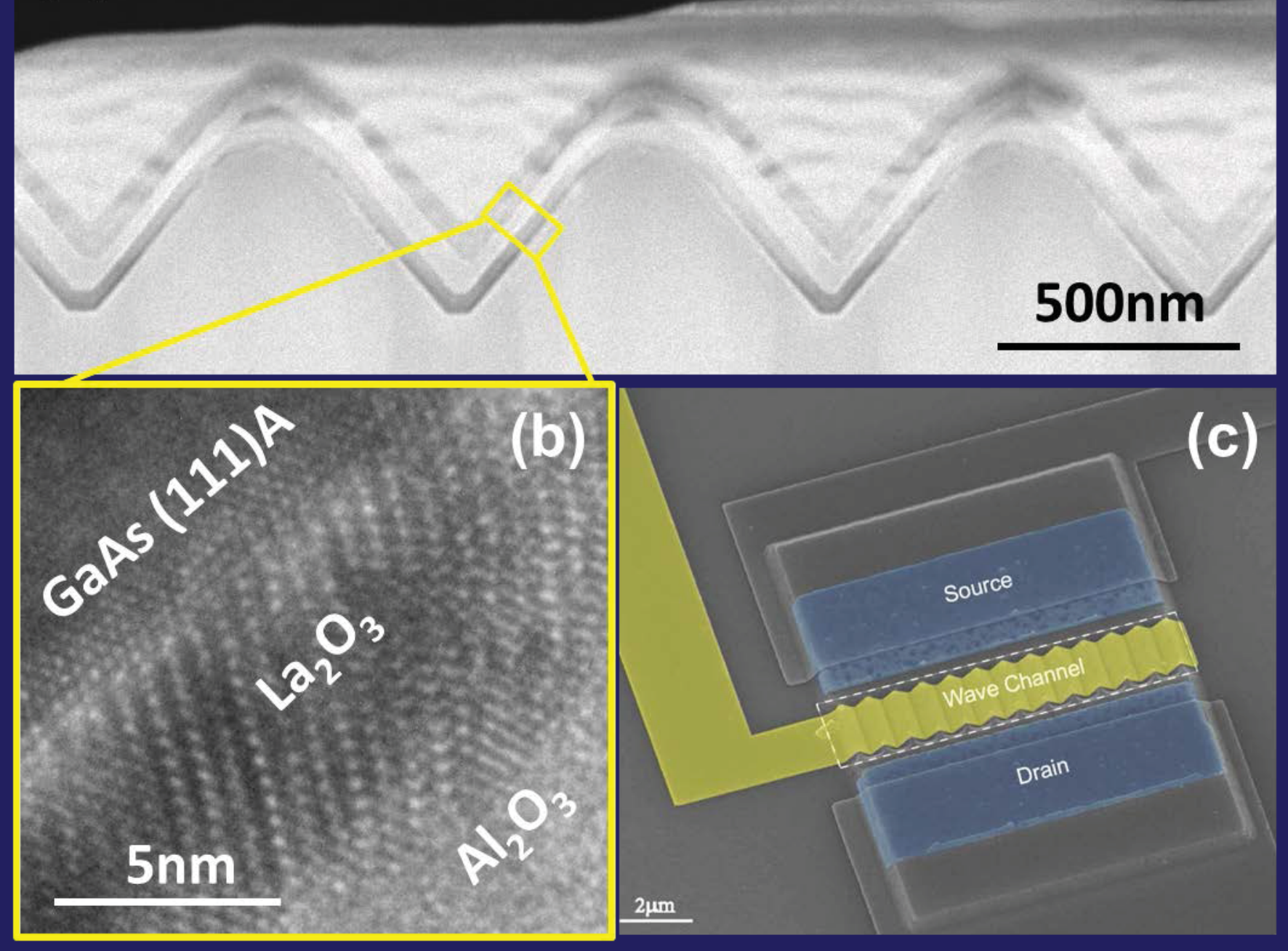




\section{Orientation to Form GaAs(111) Facets}
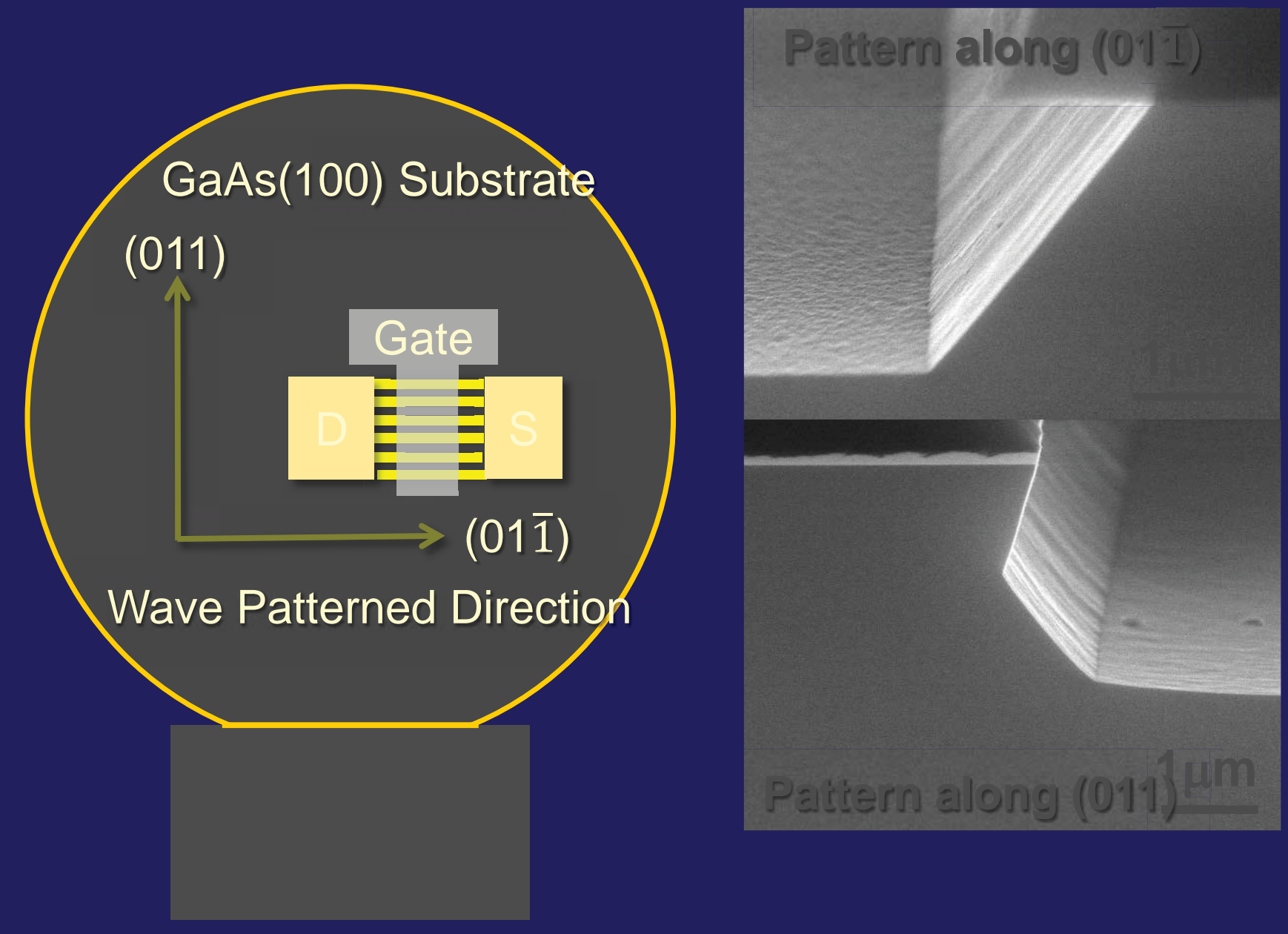


\section{WaveFET Structure on GaAs(100)}

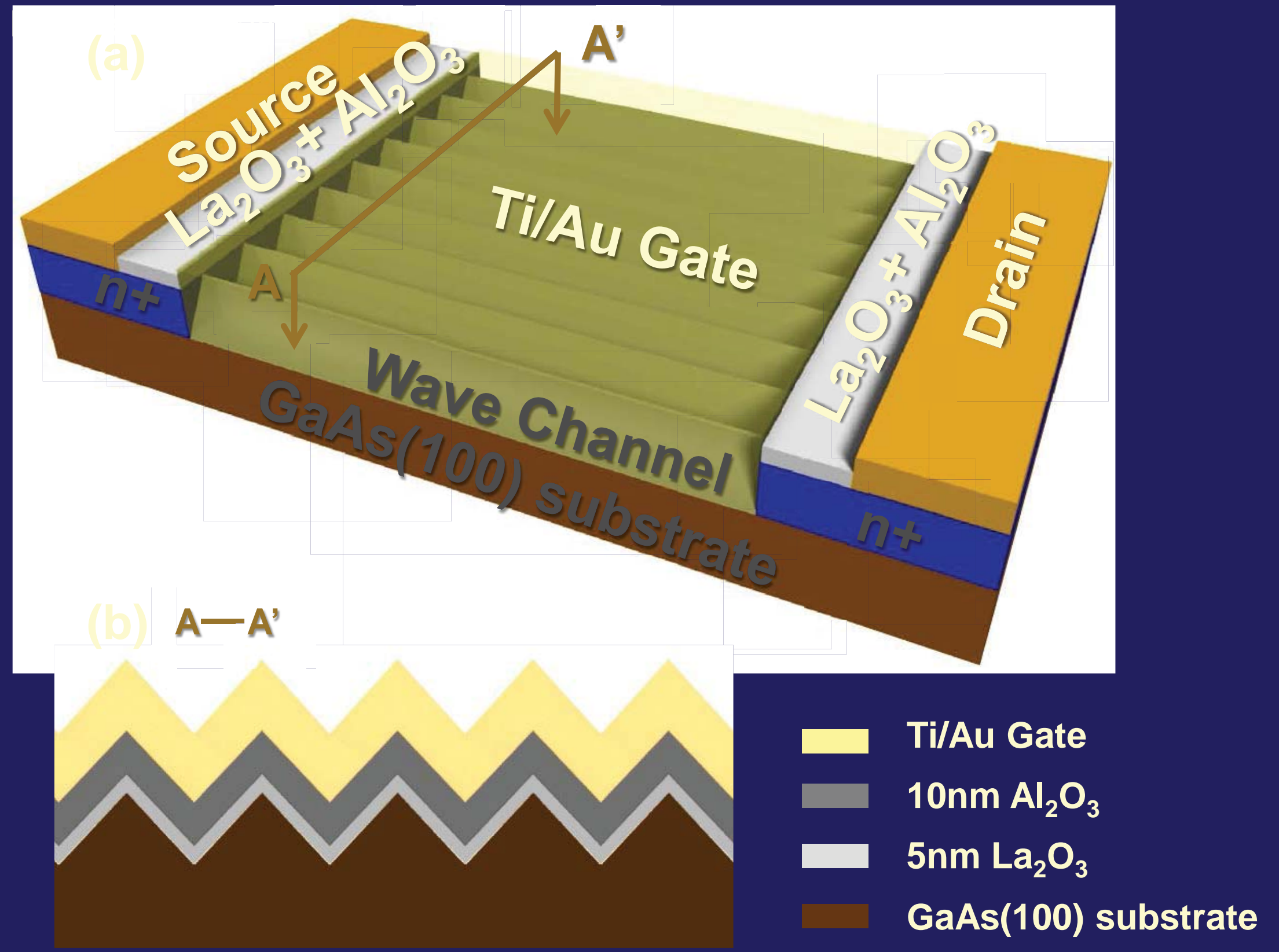




\section{WaveFET GaAs Transistor Performance}
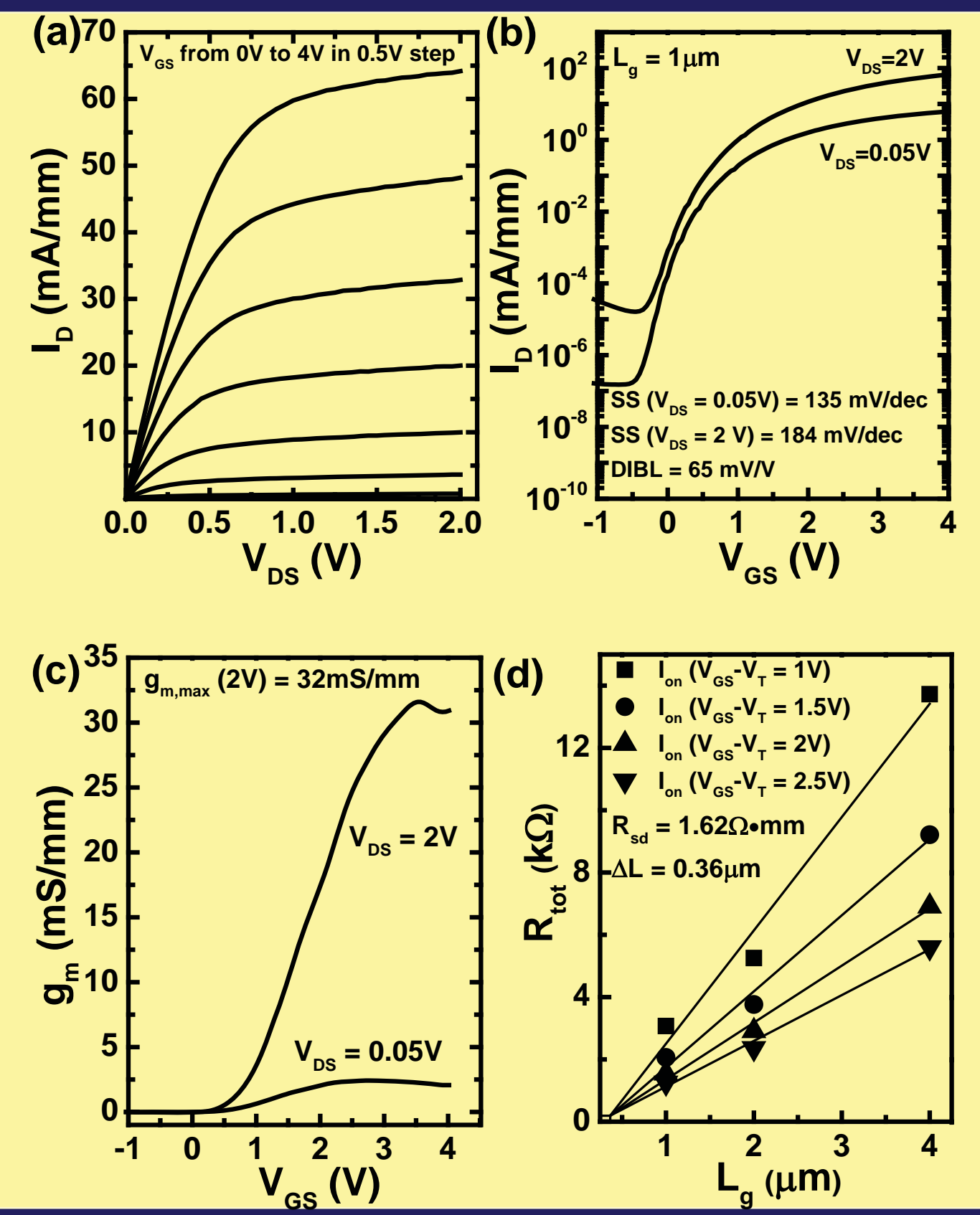


\section{Epitaxy of $\mathrm{La}_{1.7} \mathrm{Y}_{0.3} \mathrm{O}_{3}$ on $\mathrm{Ge}(111)$}

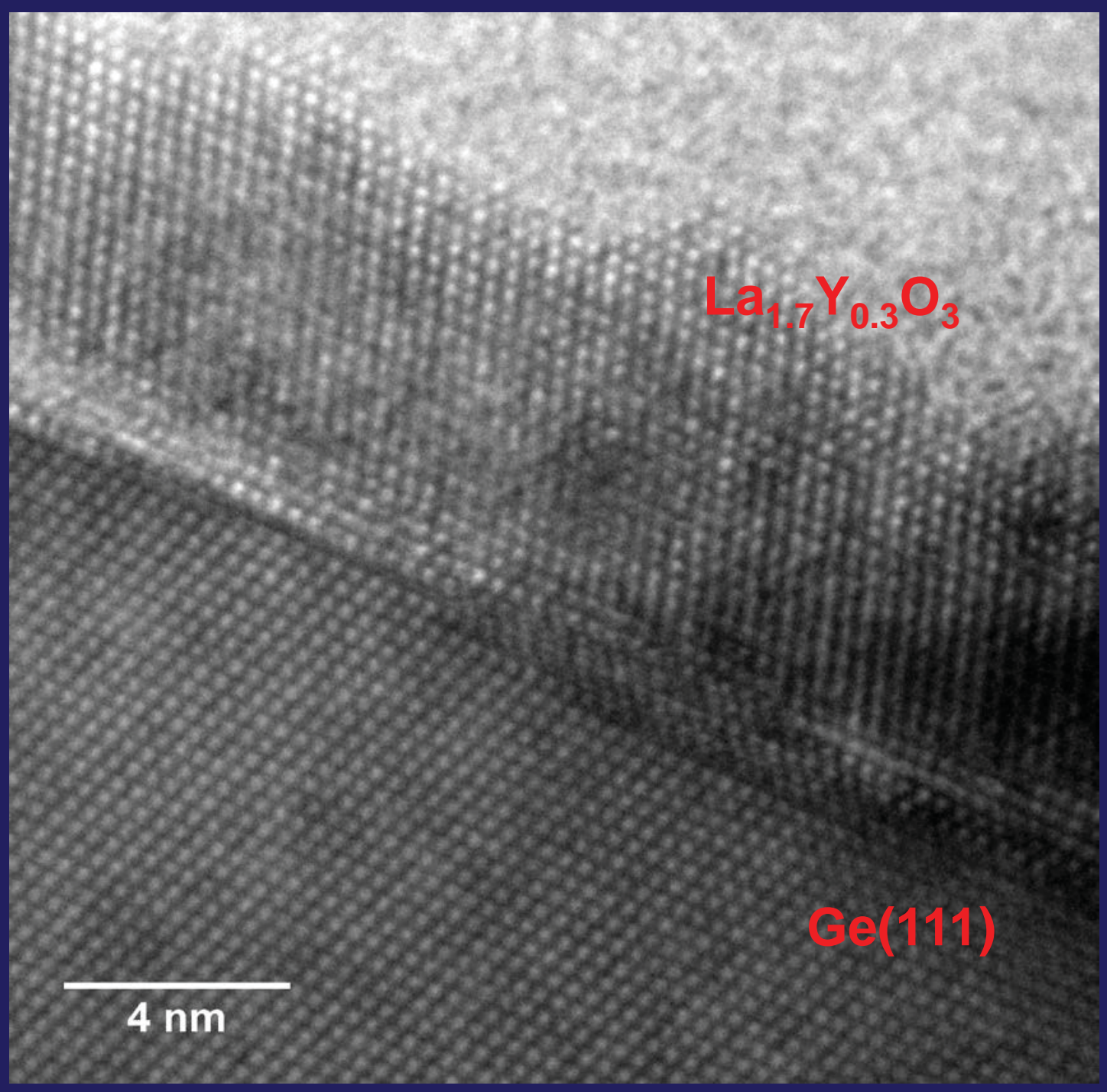




\section{Capacitors with $(\mathrm{La}, \mathrm{Y})_{2} \mathrm{O}_{3}$ on $\mathrm{Ge}(111)$}

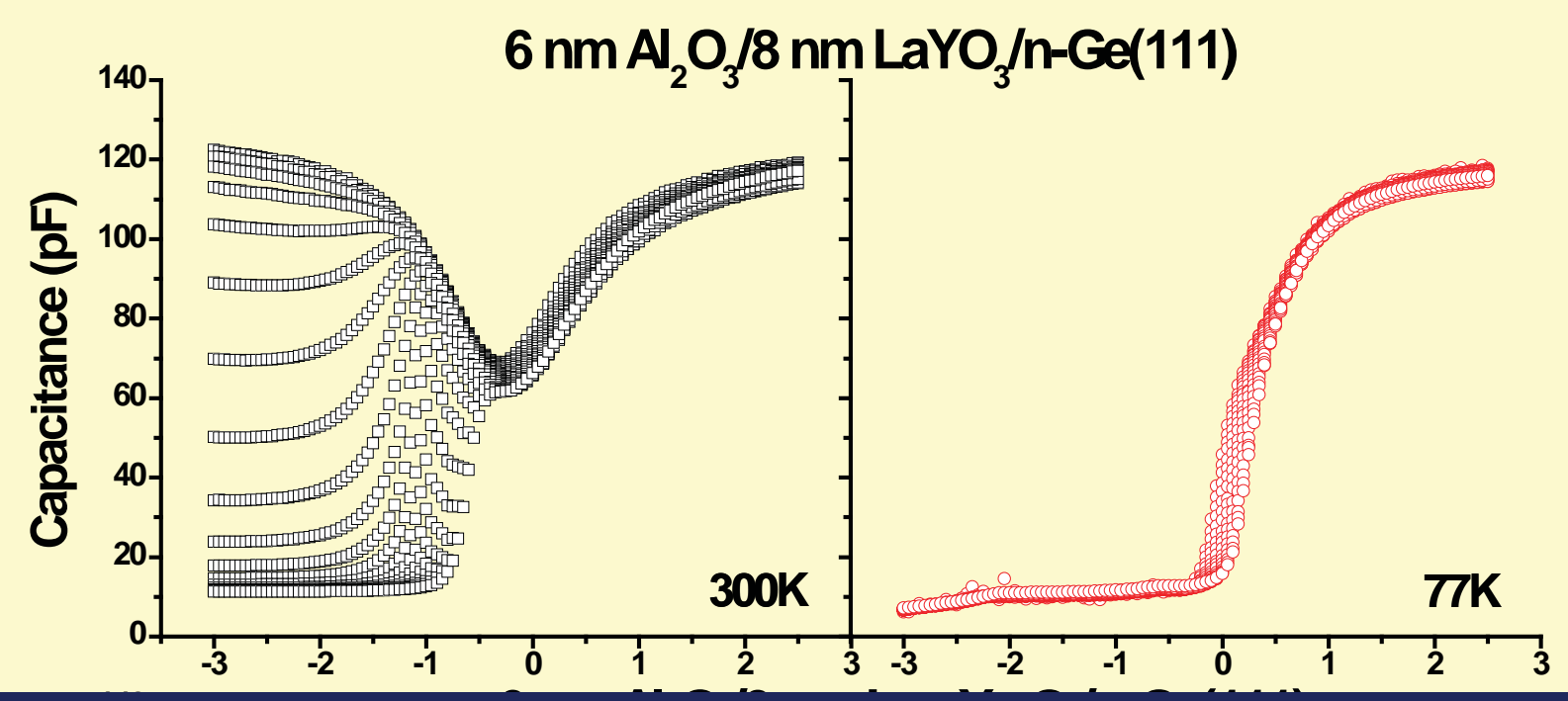

High-performance pMOS transistors are expected using this epitaxial interface 


\section{Possible Epitaxial Oxides for GaN}

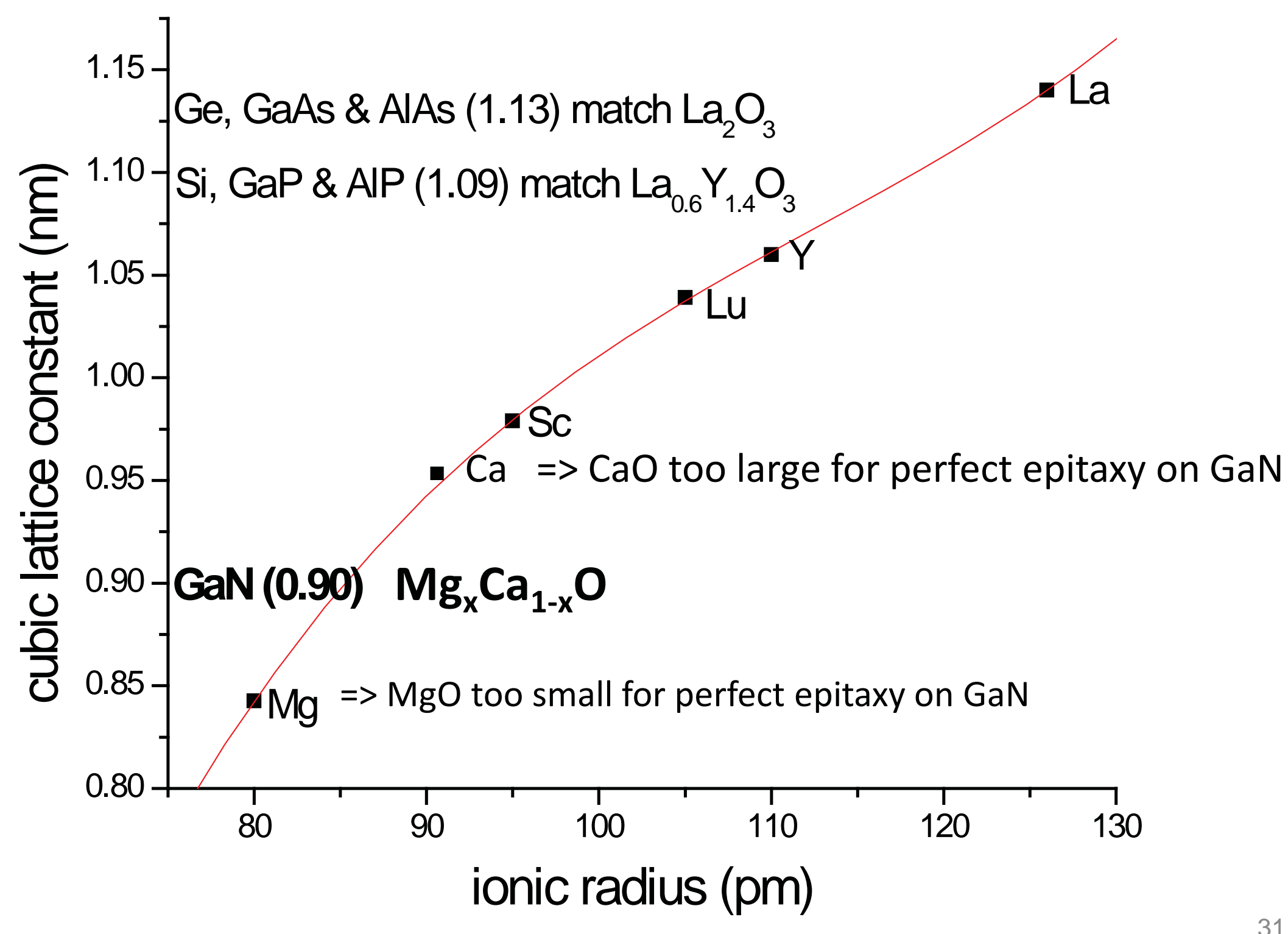




\section{Phase Diagram of $(\mathrm{Ca}, \mathrm{Mg}) \mathrm{O}$}

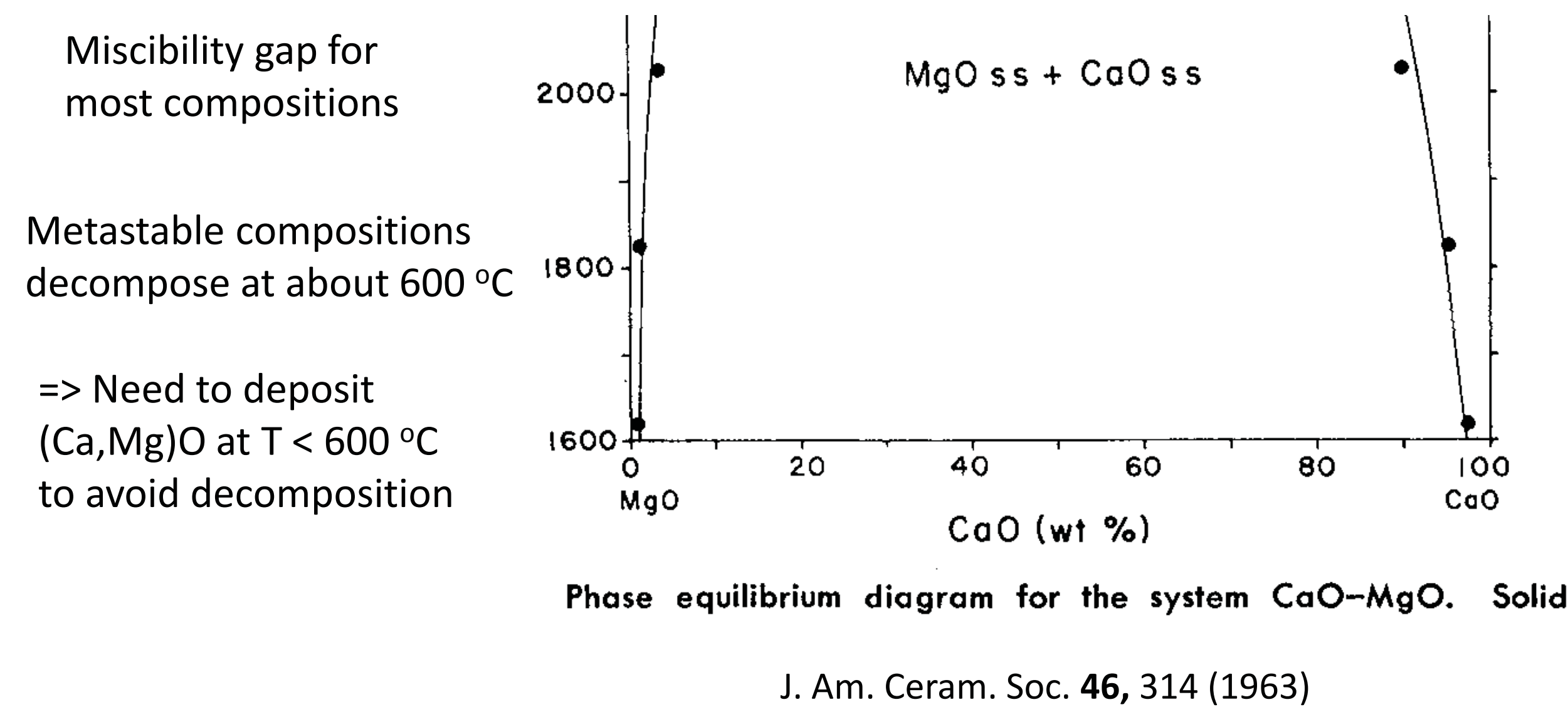




\section{Precursors for ALE of Magnesium Oxide, MgO}

bis $\left(N, N^{\prime}\right.$-di-sec-butylacetamidinato) magnesium

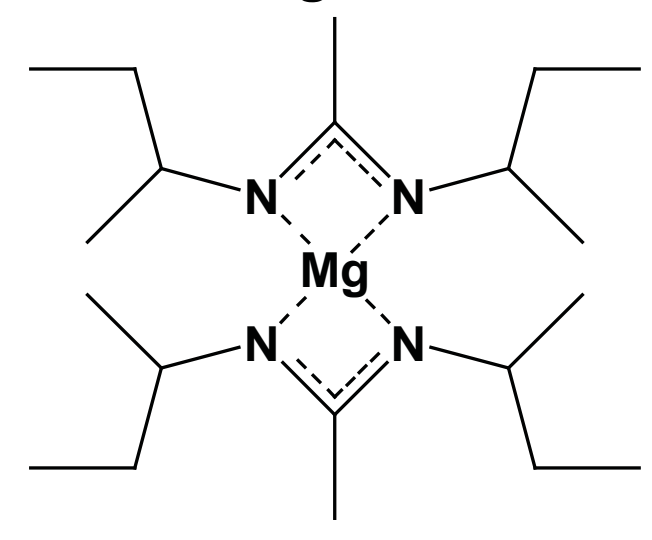

Liquid bis( $N, N^{\prime}$-di-tert-butylacetamidinato) magnesium

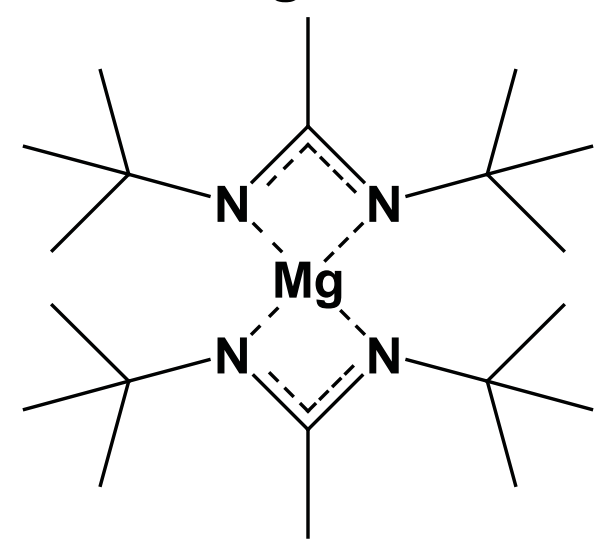

Solid

ALD bubbler temperatures $90-110^{\circ} \mathrm{C}$

Residue $<1 \%$ (clean evaporation)

Chose the liquid on left because of its ready purification by distillation and easier handling ALD of $\mathrm{MgO}$ at $300^{\circ} \mathrm{C}$, low enough to avoid thermal decomposition of (Ca, $\left.\mathrm{Mg}\right) \mathrm{O}$ 


\section{Precursors for ALE of Calcium Oxide}

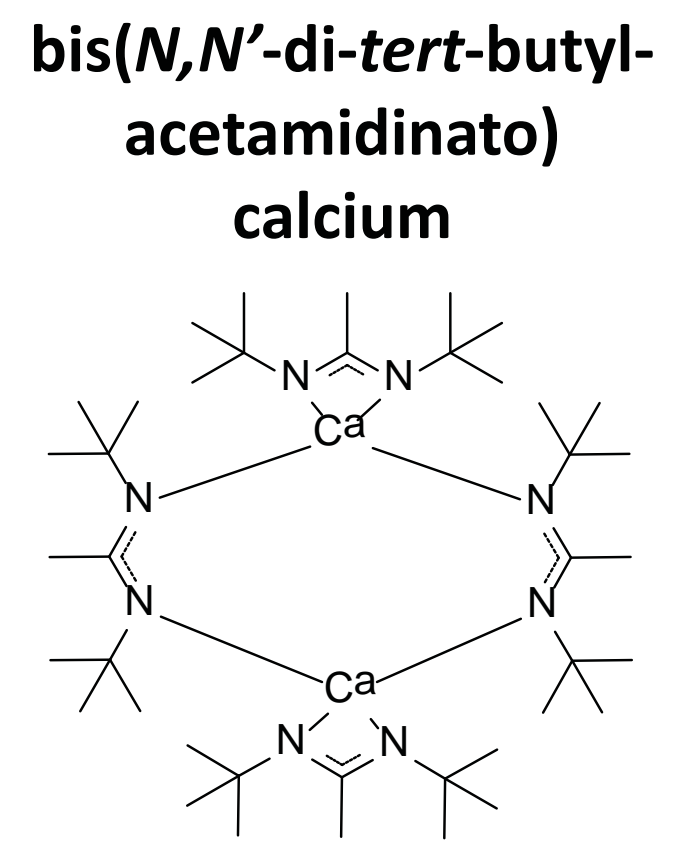

Sublimes at $185-190^{\circ} \mathrm{C}$

Low volatility

Reactive to $\mathrm{H}_{2} \mathrm{O}$
$\operatorname{bis}\left(N, N^{\prime}\right.$-diisopropylacetamidinato)

calcium

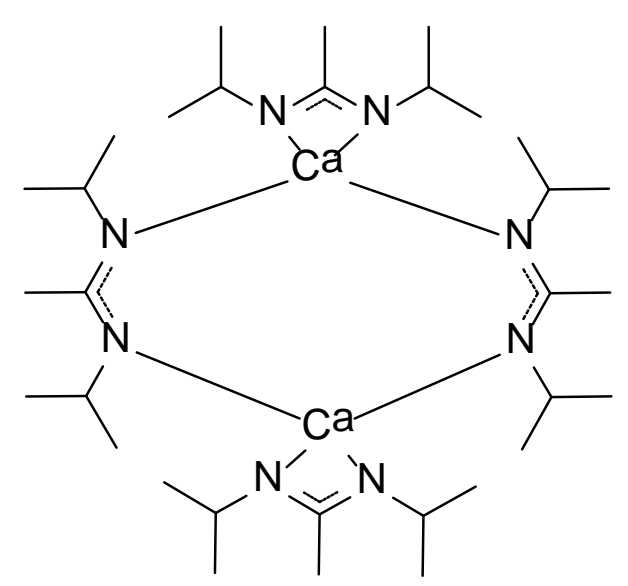

Sublimes at $135-140^{\circ} \mathrm{C}$

Higher volatility

Reactive to $\mathrm{H}_{2} \mathrm{O}$ bis( $N, N^{\prime}$-diisopropyl-

formamidinato)

calcium

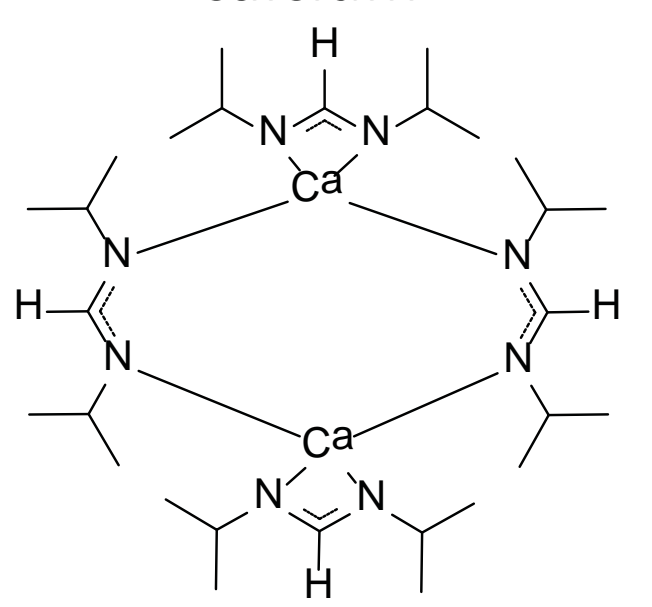

Sublimes at $95-100^{\circ} \mathrm{C}$

Superior precursor:

Highest volatility

Reactive to $\mathrm{H}_{2} \mathrm{O}$

Scaled up production

ALD of $\mathrm{CaO}$ at $300^{\circ} \mathrm{C}$, low enough to avoid decomposition of (Ca,Mg)O 


\section{Electron Diffraction from Epitaxial (Ca,Mg)O on $\mathrm{GaN}(0001)$}

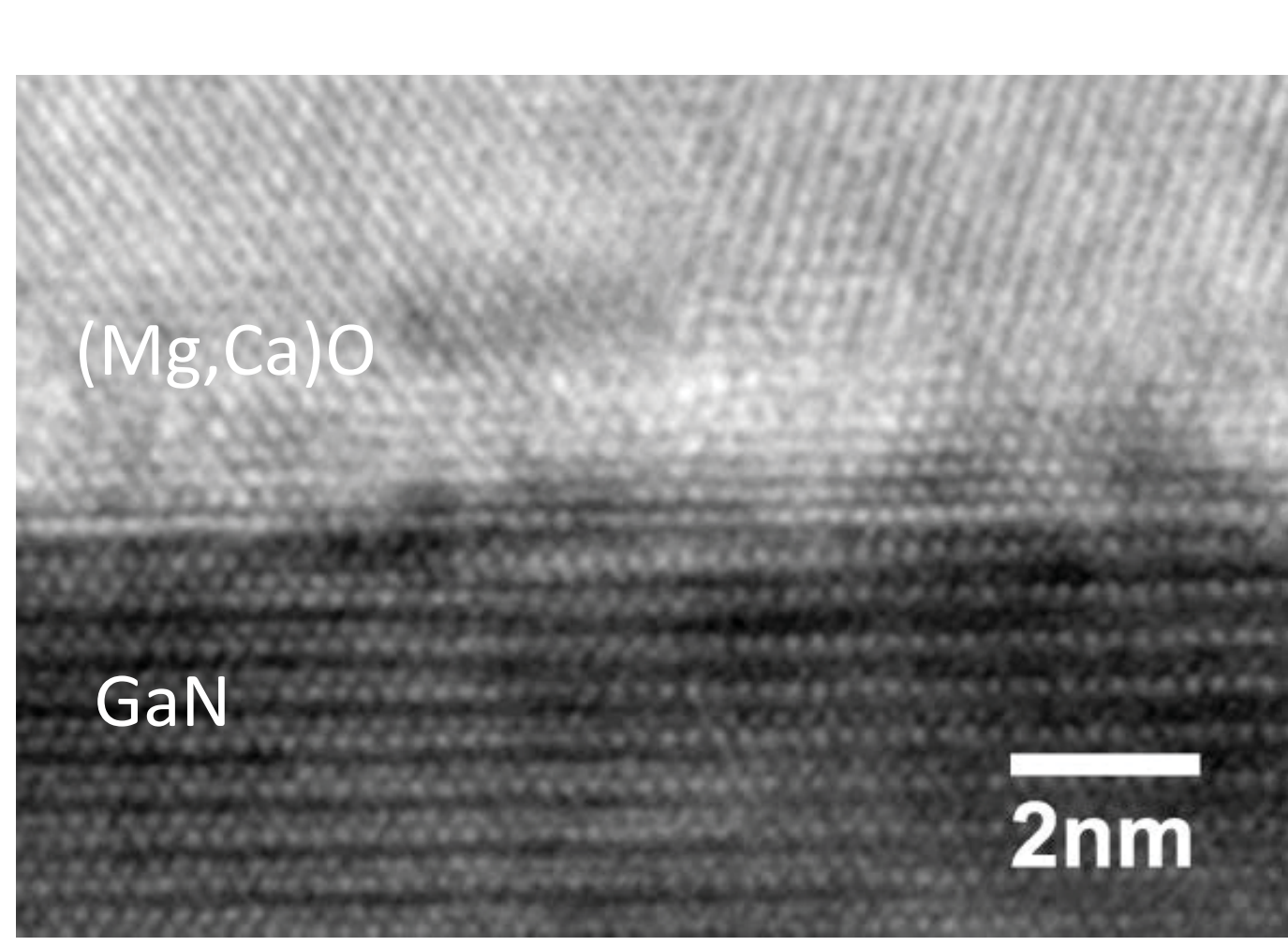

Epitaxial orientation :

(Ca,Mg)O(111) // GaN (0001)

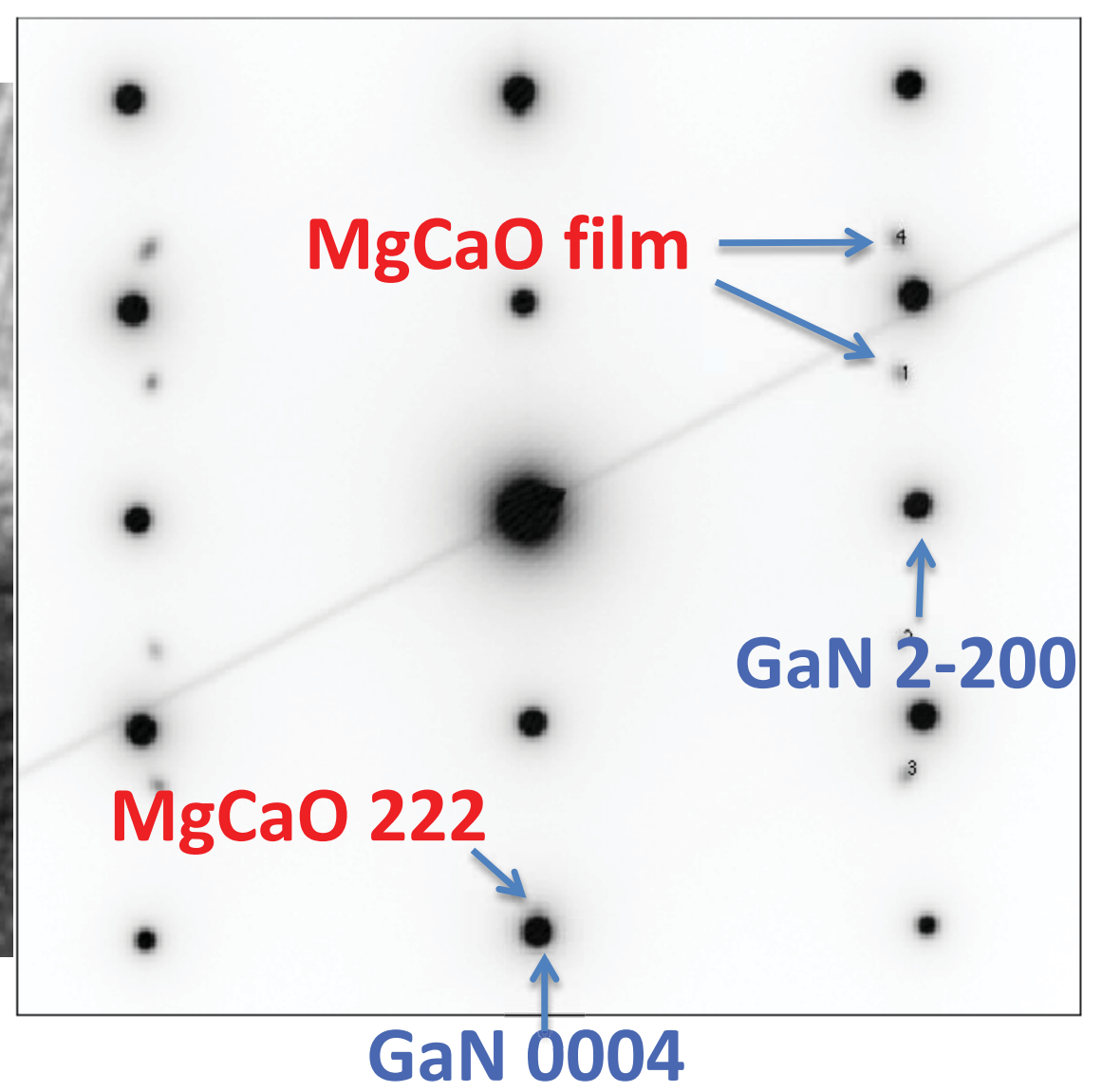

Beam Direction [11-20] 


\section{Composition of $(\mathrm{Ca}, \mathrm{Mg}) \mathrm{O}$ by RBS on Carbon Substrates}

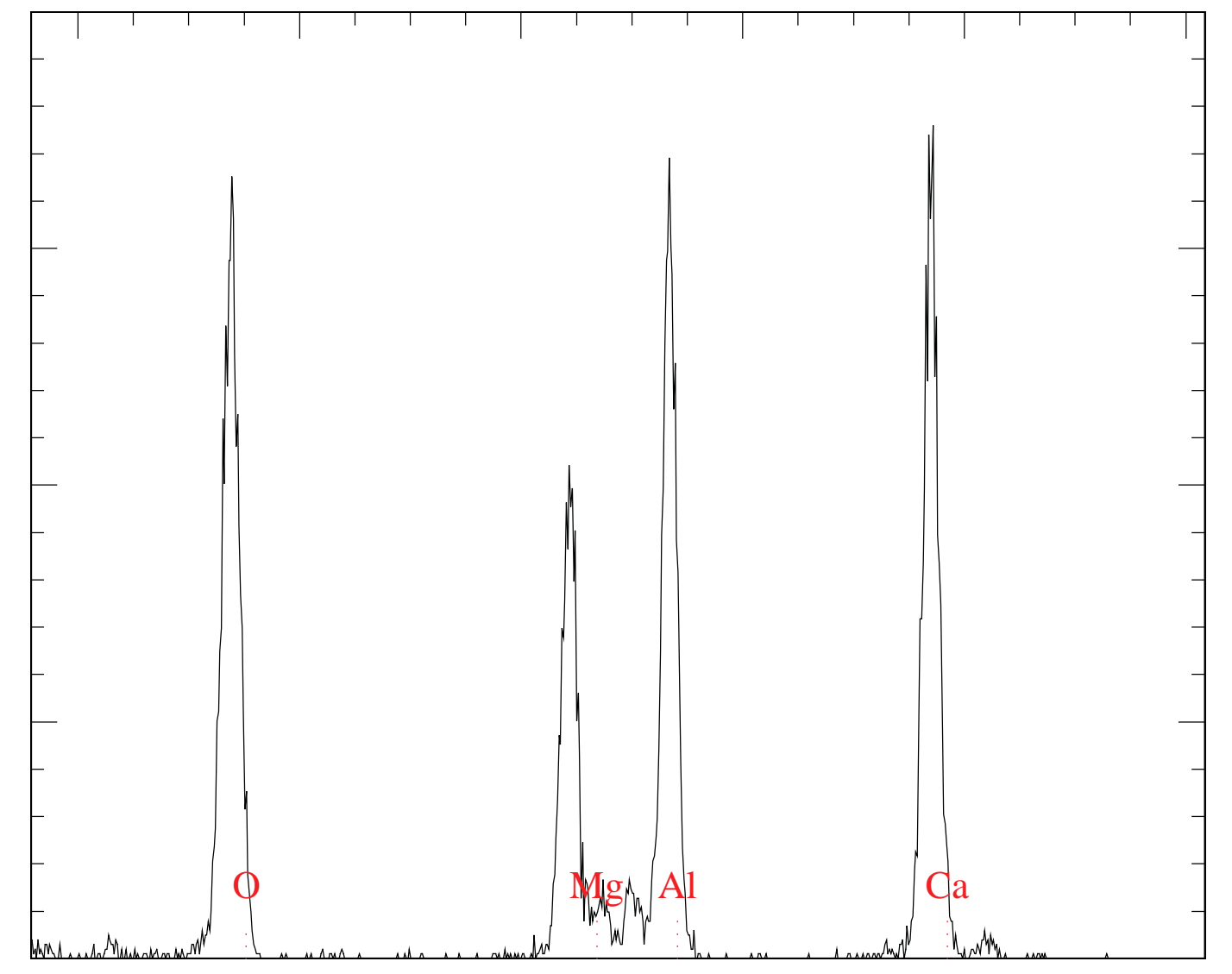

Aluminum in capping layer of $\mathrm{ALD} \mathrm{Al}_{2} \mathrm{O}_{3}$ 


\section{Lattice Constant Mismatch}

Dosing ratio $\mathrm{Mg}: \mathrm{Ca}=1: 3 ; \quad \mathrm{RBS}: \mathrm{Mg}: \mathrm{Ca}=1: 3$

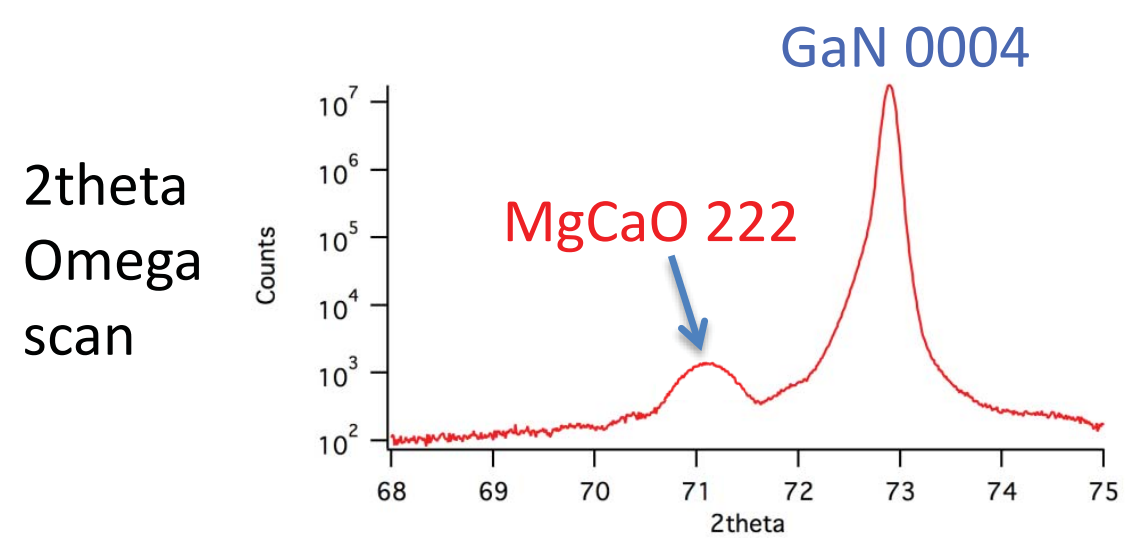

too much $\mathrm{Ca}$ for perfect lattice match

2theta $=71.09 \mathrm{~d}=1.325 \mathrm{~nm}$ mismatch $=2.2 \%$

(Vegard's law prediction: mismatch $=3.7 \%$ )

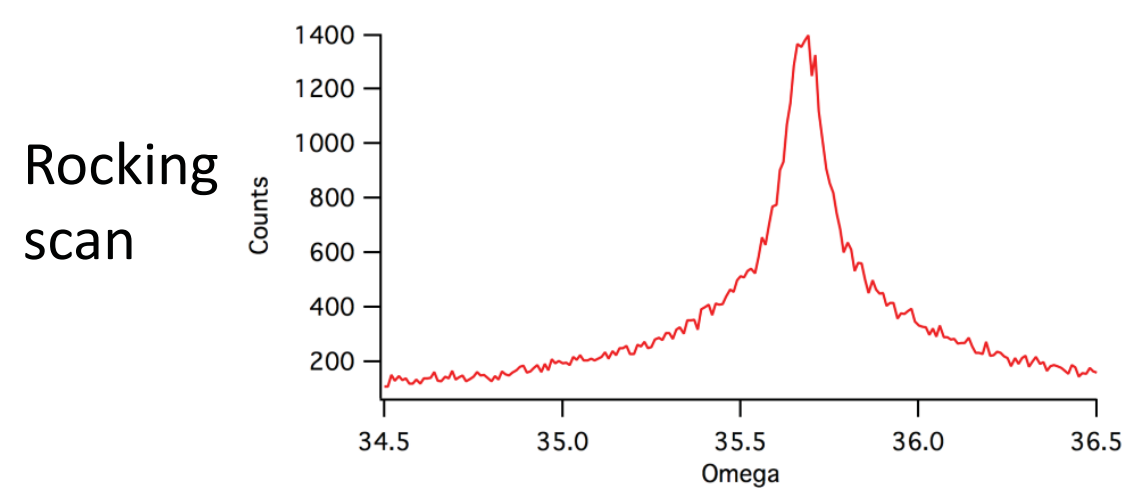

FWHM=0.23deg 


\section{$(\mathrm{Ca}, \mathrm{Mg}) \mathrm{O}$ is a good insulator on $\mathrm{GaN}$ or InAIN}

Large band offsets for both conduction and valence bands of $\mathrm{MgO}$

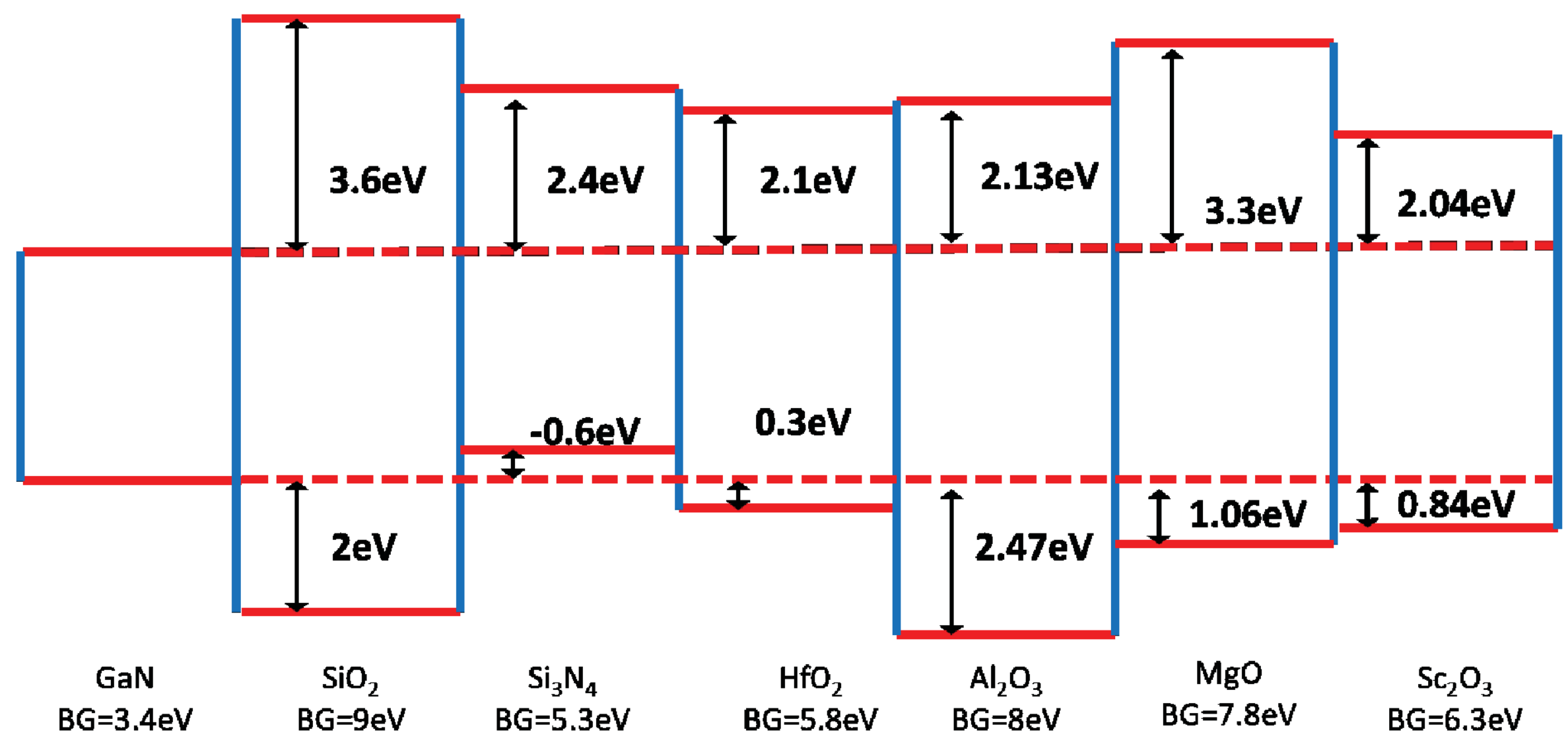

Materials 2012, 5, 1297-1335 


\section{Capacitor with $(\mathrm{Mg}, \mathrm{Ca}) \mathrm{O}$ on InAIN => Very Few Traps}

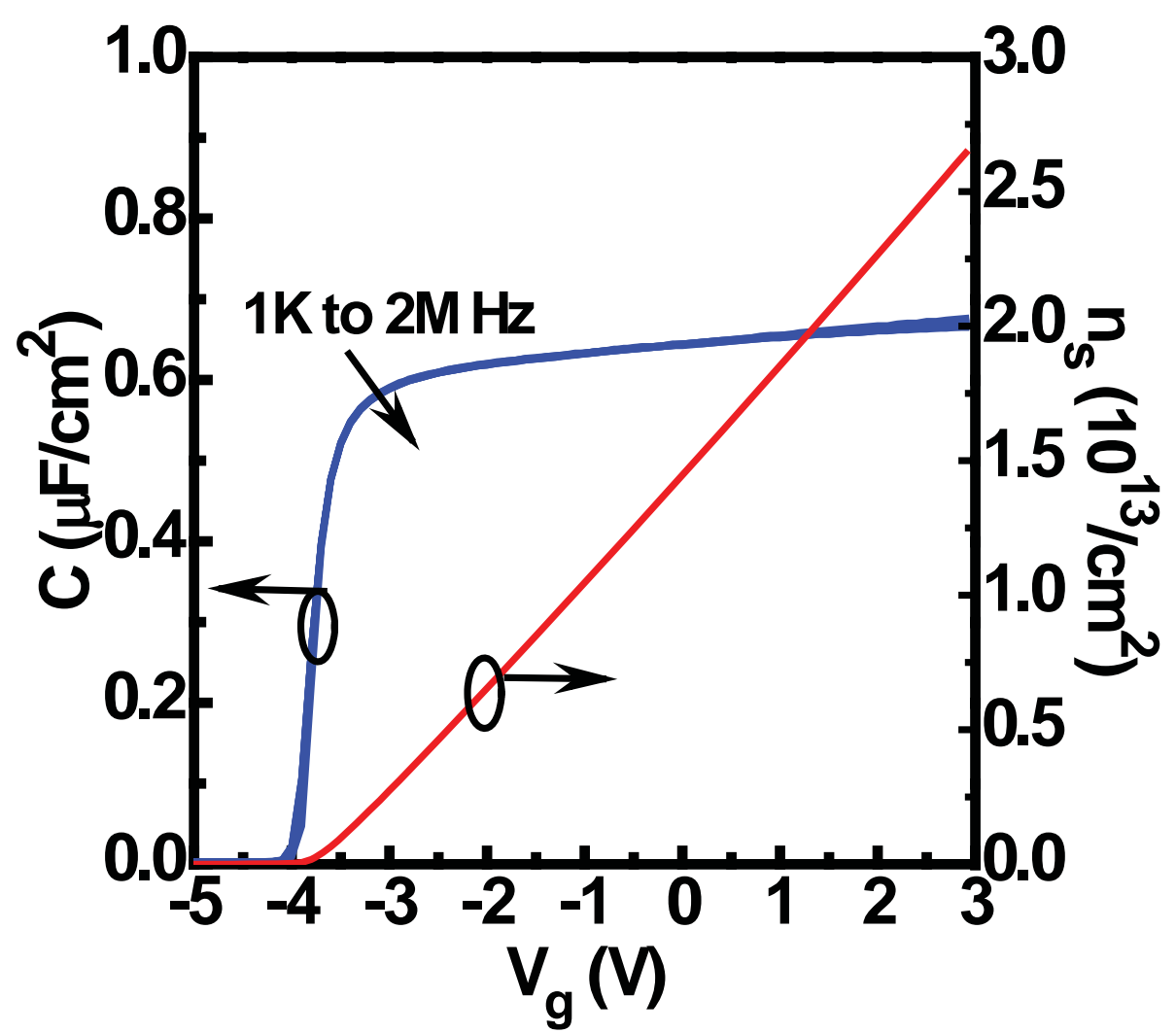

Almost no frequency dispersion

$=>$ few states at the interface.

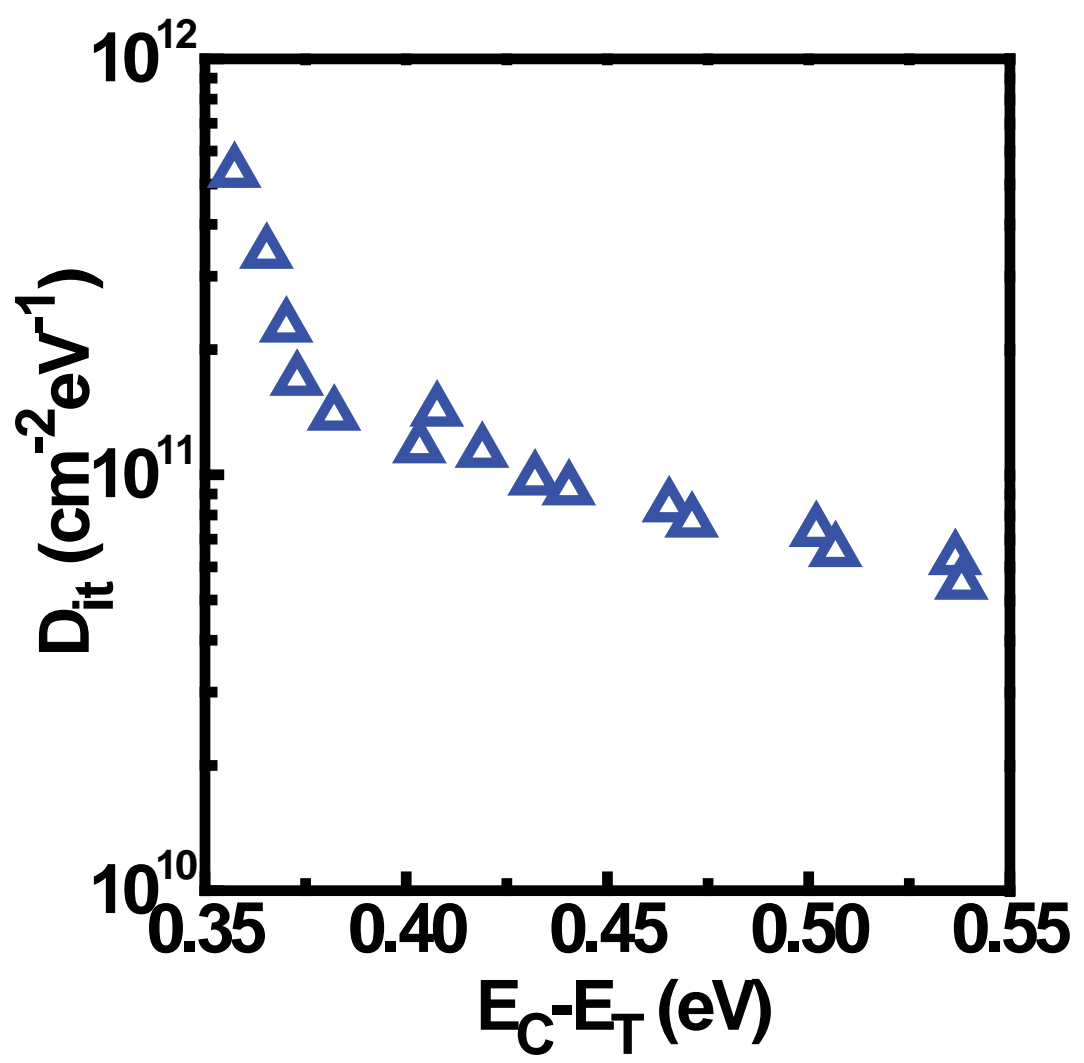

AC conductance extracts very low $D_{\text {it }}$ in the bandgap, around $10^{11} \mathrm{~cm}^{-2}$ 


\section{Drain Current vs. Gate Voltage for InAIN Transistors}

The $(\mathrm{Mg}, \mathrm{Ca}) \mathrm{O}$ gate insulator reduced the gate leakage by four orders of magnitude, from $10^{-8}$ to $10^{-12} \mathrm{~A} / \mathrm{mm}$.

The subthreshold slope is nearly ideal at $64 \mathrm{mV} / \mathrm{dec}$.

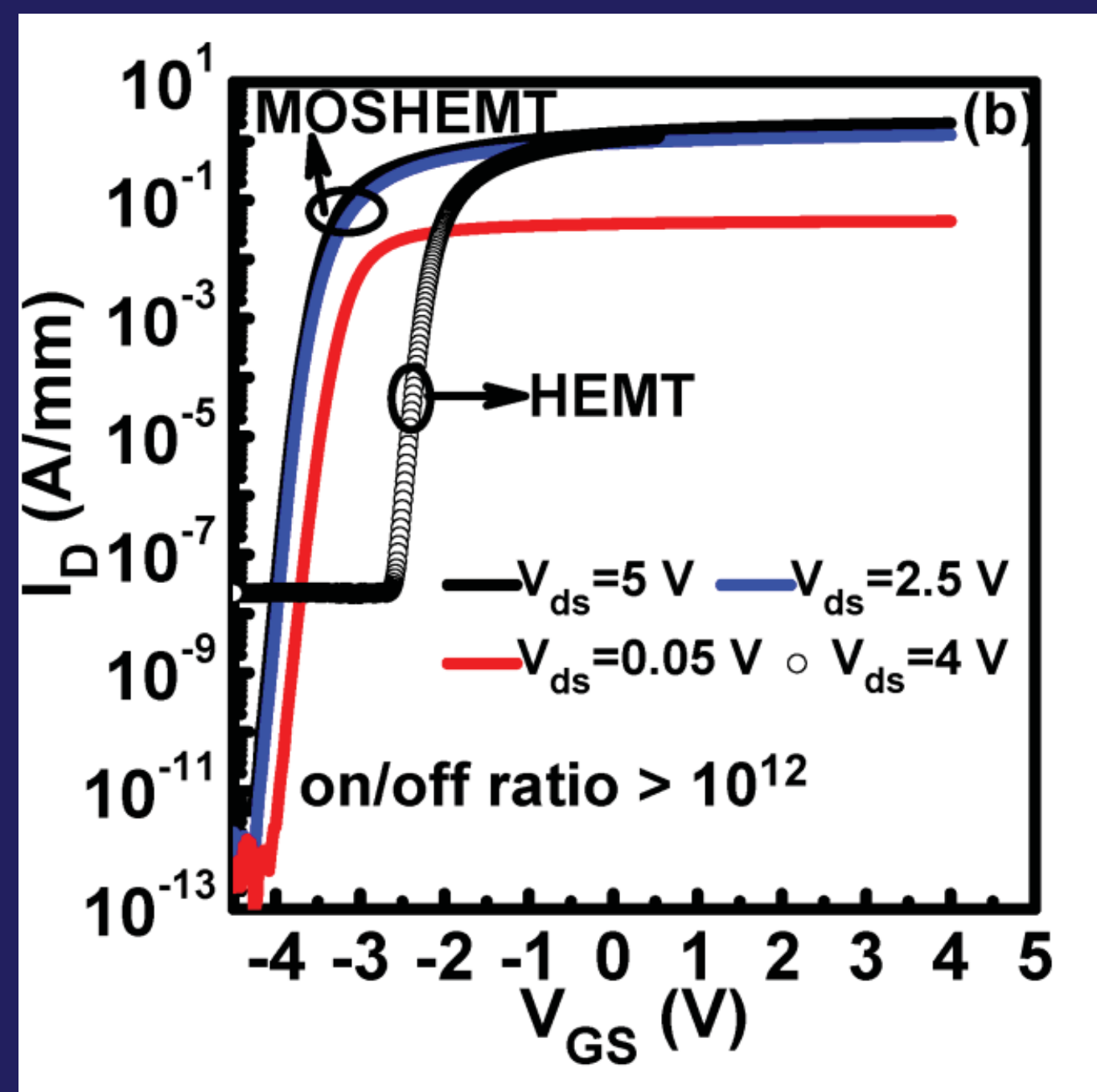




\section{Summary}

Atomic Layer Epitaxy (ALE) forms high-quality epitaxial interfaces

ALE $\mathrm{La}_{2} \mathrm{O}_{3} / \mathrm{GaAs}(111)=>$ both $p$ - and $n$ - type capacitors with low dispersion $=>$ the first CMOS circuits with both channels in GaAs

ALE $\mathrm{La}_{2} \mathrm{O}_{3} / \mathrm{Ge}(111)=>$ epitaxial structures

ALE $(\mathrm{Mg}, \mathrm{Ca}) \mathrm{O} / \mathrm{GaN}$ or InAIN => capacitors with low dispersion

ALE $(\mathrm{Mg}, \mathrm{Ca}) \mathrm{O} / \mathrm{InAIN}=>$ transistors with low gate leakage

ALE is a scalable process for making high-performance devices 


\section{Harvard Acknowledgements}

Yiqun Liu(Global Foundries), Xinwei Wang (PKU), Xiabing Liu, Sang Bok Kim

Precursors: Dow Chemical Company

Analyses: Applied Materials, IBM, IMEC

Facilities at Harvard's Center for Nanoscale Systems (CNS), a member of the National Nanotechnology Infrastructure Network (NNIN), supported by the US National Science Foundation
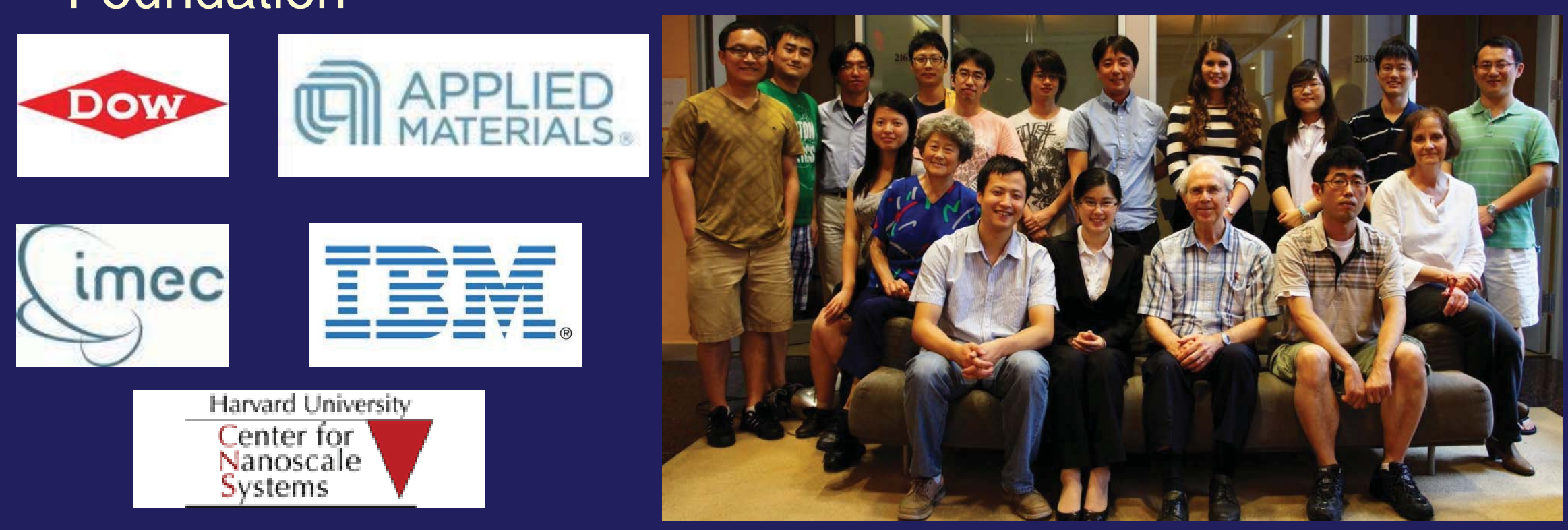


\section{Purdue Acknowledgements}

Purdue University: P. D. Ye, J.J. Gu (Intel), Y.Q. Wu (IBM), Y. Xuan, J.Y. Zhang,

L. Dong, M. Xu (AMAT), H.C. Lin (IMEC),

Kun Xu (NIST), R.S. Wang (PKU), C. Wang (Fudan),

T. Shen (NIST), D. Varghese(Intel), M.A. Alam,

M. Lundstrom,

SRC FCRP: D.A. Antoniadis (MIT), R.M. Wallace (UTD),

A. Kummel (UCSD), S. Oktyabrsky (SUNY Albany),

J. del Alamo (MIT)

IQE: W.K. Liu, J.M. Fastenau, D. Lubyshev

ASM: G.D. Wilk SRC: K.K Ng NTU: M. Hong and J. Kwo

Harvard University: Y.Q. Liu (GlobalFoundries), X.W. Wang (PKU), X. Liu,

R.G. Gordon

Lehigh University: W.K. Wang (IBM), J.C.M. Hwang (AFOSR)

Fudan: M.F. Li and D.M. Huang

U. Padova: N. Wrachien, E. Zanoni, G. Meneghesso

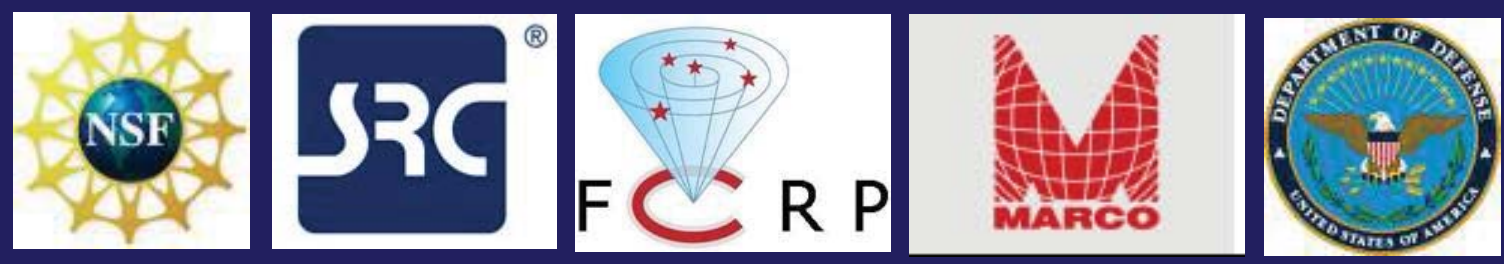

